\title{
WestVirginiaUniversity
}

THE RESEARCH REPOSITORY @ WVU

Graduate Theses, Dissertations, and Problem Reports

2014

\section{Psychological Measures to Predict Serious Prison Violence}

\author{
Allison M. Schenk \\ West Virginia University
}

Follow this and additional works at: https://researchrepository.wvu.edu/etd

\section{Recommended Citation}

Schenk, Allison M., "Psychological Measures to Predict Serious Prison Violence" (2014). Graduate Theses, Dissertations, and Problem Reports. 629.

https://researchrepository.wvu.edu/etd/629

This Dissertation is protected by copyright and/or related rights. It has been brought to you by the The Research Repository @ WVU with permission from the rights-holder(s). You are free to use this Dissertation in any way that is permitted by the copyright and related rights legislation that applies to your use. For other uses you must obtain permission from the rights-holder(s) directly, unless additional rights are indicated by a Creative Commons license in the record and/ or on the work itself. This Dissertation has been accepted for inclusion in WVU Graduate Theses, Dissertations, and Problem Reports collection by an authorized administrator of The Research Repository @ WVU.

For more information, please contact researchrepository@mail.wvu.edu. 
Psychological Measures to Predict Serious Prison Violence

\author{
Allison M. Schenk, M.S. \\ Dissertation submitted \\ to the Eberly College of Arts and Sciences \\ at West Virginia University \\ in partial fulfillment of the requirements for the degree of \\ Doctor of Philosophy in \\ Psychology \\ William Fremouw, Ph.D., ABPP, Chair \\ Nicholas Bowman, Ph.D. \\ Thomas Horacek, Ph.D. \\ Kevin Larkin, Ph.D. \\ Aaron Metzger, Ph.D. \\ Natalie Shook, Ph.D. \\ Department of Psychology
}

Morgantown, West Virginia

2014

Keywords: Prison Violence, Psychological Measures, Mental Health History, MMPI-2-RF, Substance Abuse, Intelligence, Violence Severity Index

Copyright 2014 Allison M. Schenk 


\title{
ABSTRACT \\ Psychological Measures to Predict Serious Prison Violence
}

\author{
Allison M. Schenk
}

With 2.2 million adults incarcerated throughout the United States (Glaze \& Herberman, 2013), prisons are crowded, volatile environments susceptible to violence. Prior research has identified demographic and criminal variables that consistently predict prison violence. The purpose of this study was to investigate the utility of psychological variables to predict prison violence among 180 inmates from a state prison in West Virginia. The psychological variables studied included history of mental illness and results from psychological assessments (Beta-III, MMPI-2-RF, TCU Drug Screen II). Using a logistic regression analysis, history of mental illness and the MMPI-2-RF scale of Psychoticism accurately predicted violent inmates from non-violent inmates while controlling for demographic and criminal variables. A weighted measure of violence severity was calculated and a multiple regression analysis was conducted. In the final model, mental health history significantly predicted Violence Severity Index scores. Hierarchical cluster analysis identified three meaningful groups of violent inmates based on the five MMPI-2RF personality psychopathology scales. Eleven inmates were characterized by high scores on Psychoticism and Negative Emotionality/Neuroticism. Another cluster of 15 inmates were defined by high scores on the Aggressiveness and Disconstraint (e.g., risk-taking, impulsivity) scales. The third group had low scores on these four scales and had moderate scores on Low Positive Emotionality/Introversion. The results of this study support the conclusion that psychological variables have utility in predicting prison violence, but more research is needed to continue understanding this relationship. 


\section{Acknowledgements}

I would first like to thank my graduate advisor and dissertation committee chairperson, William Fremouw, who has tirelessly supported and encouraged me for the past five years. Bill has gone out of his way to provide me with invaluable opportunities that have shaped me into the professional I am today. I consider myself incredibly fortunate to have Bill as an advisor and cannot imagine this journey without him. Thank you does not seem adequate to truly capture the gratitude I feel for having Bill as an advisor and a friend.

In addition, I would like to acknowledge my dissertation committee member, Thomas Horacek, for facilitating the approval and implementation of this study with the West Virginia Division of Corrections. I also want to thank Nicholas Bowman, Kevin Larkin, Aaron Metzger, and Natalie Shook for their time and meaningful contributions they provided as part of my dissertation committee. I would like to express my gratitude to my colleagues and friends at West Virginia University who have made graduate school an enjoyable and memorable experience. I am especially appreciative of Colleen Keelan for her company on the long trips to Huttonsville, West Virginia, her willingness to code and collect data, and her uplifting attitude. It has been a pleasure to work together and I look forward to future collaborations throughout our careers.

Most importantly, I would like to thank my parents who have always believed in me. I never could have done this without their constant support and encouragement. I realize the sacrifices they have made to give me every opportunity in life and I am so incredibly grateful. They are the most wonderful parents and role models a person could ever dream of having, and I am extraordinarily blessed to have them as my parents. I love you so much, Mom and Dad (and Bucky too)! 
Table of Contents

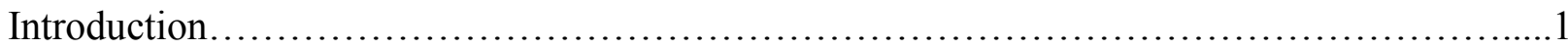

Demographic Variables.....................................................5

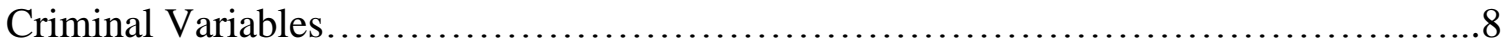

Psychological Variables.....................................................9

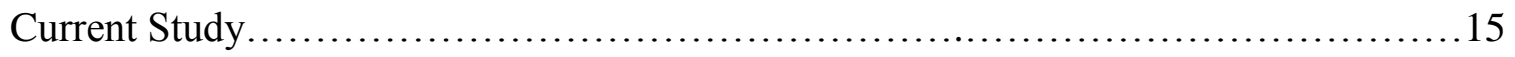

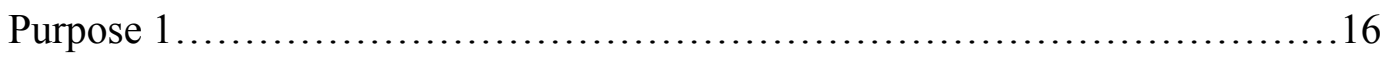

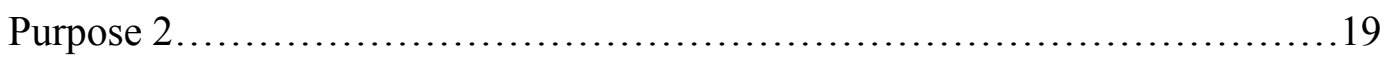

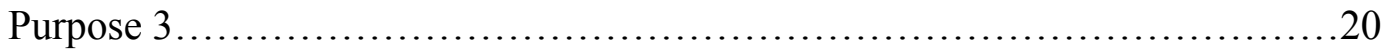

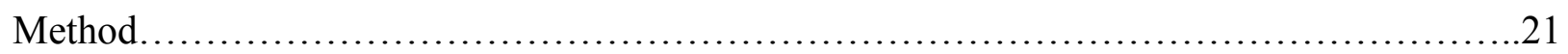

Participants............................................................. 21

Materials...............................................................24

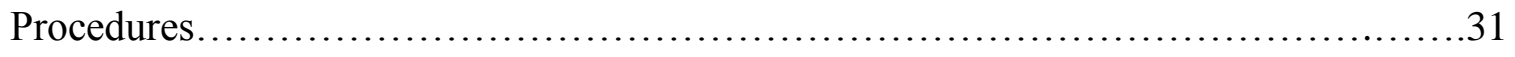

Reliability Procedures........................................................ 31

Results...................................................................... 32

Demographic and Univariate Analyses........................................ 32

Purpose 1 -Logistic Regression Results....................................36

Purpose 2-Hierarchical Multiple Regression Results............................ 38

Purpose 3-Cluster Analysis of Violent Inmates............................... 39

Prototypes of Clusters..............................................41

Discussion................................................................... 43 
Non-Statistically Significant Findings ........................................ 45

Limitations.................................................................. 47

Study Implications and Future Directions...................................49

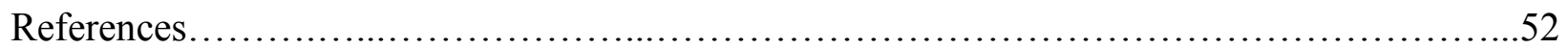

Tables......................................................................63

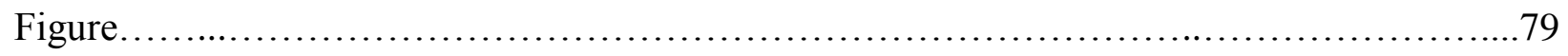

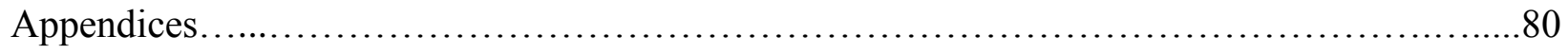

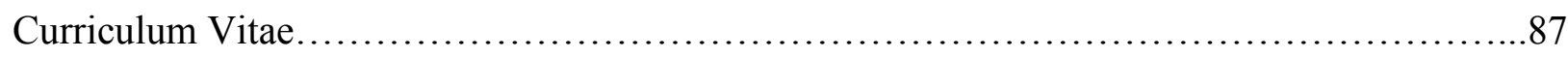




\section{Psychological Measures to Predict Serious Prison Violence}

At a rate of 920 per 100,000, the United States incarcerates more people per capita than any other country in the world (Glaze \& Herberman, 2013). In comparison, Russia’s incarceration rate is 577 per 100,000 and our neighboring country of Canada has an incarceration rate of only 117 per 100,000 people (International Centre for Prison Studies, 2010). By the end of 2012, over 2.2 million individuals were incarcerated in the United States (Glaze \& Herberman, 2013). Specifically, 1,267,000 inmates were incarcerated in state prisons and 216,900 inmates were held in federal correctional facilities. The remainder of these inmates were incarcerated in jails and privately-operated prisons. When rates of community supervision (including probation and parole) were combined with the population of individuals held in jail and prison facilities (state, federal, and privately-operated) in the United States in 2012, it amounted to $6,937,600$ individuals that were involved with the adult correctional system. This rate is approximately $2.9 \%$ of the country's total population and amounts to one in every 35 adults having involvement with the correctional system (Glaze \& Herberman, 2013). Specifically, the state of West Virginia incarcerated 7,096 men and women throughout its prisons and jails in 2013 (Hildebrand, 2013).

With so many individuals incarcerated, there is a constant need for more prison facilities. Between 2001 and 2005, state and federal prison facilities increased from 1,668 to 1,821 with a drastic rise in private facilities accounting for the majority of this increase (Stephen, 2008). One of the reasons for this upsurge in contracting-out the management of some prisons to private companies is to help alleviate the problem of overcrowding in many existing prisons (Stephen, 2008). Given the multitude of correctional institutions, there is a great deal of variability in the security levels, policies and procedures, and conditions of prisons throughout the United States. 
West Virginia has fourteen prisons throughout the state to house 5,355 sentenced inmates (Hildebrand, 2013). These prisons vary significantly in security level, size, staff-to-inmate ratio, and the types of offenders they hold. The oldest and most diverse prison in the state is Huttons ville Correctional Center (HCC), which holds up to 1,138 male offenders of all security levels. It was estimated that in 2013, over 3,100 inmates were processed in and out of HCC (Hildebrand, 2013). The housing units at HCC vary from general population dorms to therapeutic communities of inmates participating in substance abuse or sex offender-specific treatment, as well as segregation units of solitary cells that confine inmates for 23 hours of the day.

By their nature, prisons house individuals deemed to be incapable of following the rules and regulations in order to live independently in society. It is not surprising that the combination of adult criminals from diverse backgrounds in an overcrowded setting with very little freedom often erupts in violence. On the extreme end of the prison violence spectrum, there was a rate of four homicides per 100,000 inmates in 2002, as reported by the Bureau of Justice Statics (Mumola, 2005). More recently, the rate of homicides in jails was reported at three per 100,000 inmates and five homicides per 100,000 inmates in state prison facilities throughout the country (Noonan, 2013). These rates are substantial decreases from the 54 homicides per 100,000 inmates documented in 1980 (Mumola, 2005). Although the homicide rate in society varies throughout the United States based on a multitude of factors, it was approximated that from 2011 through 2012, the homicide rate was 4.7 homicides per 100,000 people in society (Unified Crime Report, n.d.).

When other violent acts in prison (e.g., assault) are examined, the frequency is much higher. Not only are assaults more common, but it is much more likely for these altercations to 
go unreported or undetected than for a homicide to go unnoticed. This discrepancy raises the issue of examining official documentation of violence versus inmate self-report. For example, when inmates were asked to report their involvement in physical assaults in state and federal correctional facilities, 300,000 inmate-on-inmate assaults were estimated compared with 34,000 assaults that were documented in official records (Bryne \& Hummer, 2007). Similarly, selfreport data estimated a rate of $21 \%$ of inmates admitting to being the victim of a physical assault perpetrated by another inmate in the past six months across 13 prisons (Wolff, Blitz, Shi, Siegel, $\&$ Bachman, 2007). The rates of victimization self-reported by inmates also vary significantly based on how the questions are phrased (Wolff, Shi, \& Bachman, 2008). Interestingly, official rates of prison assaults in the U.S. were not published, and most information on "official" base rates of prison violence comes from individual studies of localized prisons with limited generalizability.

The Bureau of Justice Statistics did report a rate of violent inmate-on-staff assaults of 155.7 per 1,000 people (Duhart, 2001). This rate is specifically for correctional officers, and is estimated to be more than twice as high as the rate for violent attacks on mental health workers in prison settings. Stephan and Karberg (2003) reported physical assaults against staff increased by $27 \%$ from 1995 to 2000 in both state and federal institutions. Research indicates that the frequency of inmate-on-staff assaults decreases as the severity increases (Sorensen, Cunningham, Vigen, \& Woods, 2011). Attacks on staff have serious repercussions for the entire prison system ranging from impaired physical and psychological functioning of the attacked employee, as well as expenses associated with absenteeism and workman's compensation (Gadon, Johnstone, \& Cooke, 2006). There also is the potential for staff turn-over and tarnished reputation of the facility that can come from prison violence targeted at staff. 
Regardless of the exact rate, prison violence is a crucial issue that needs more attention in order to reduce the harm caused to staff and inmates. There are procedures to punish inmates that are violent while incarcerated such as transferring these inmates to more secure and restrictive prisons or moving them to punitive units such as Specialized Housing Units (SHU). However, by this time the violence has occurred, and although that particular inmate may be temporarily prevented from inflicting more violence, the damage of the original act is already done. The deterrent effect of transferring inmates or placing them in punitive segregation (e.g., SHU) for their violent acts has been argued; however, the logic of this theory is questionable. Specifically, if these individuals were successfully deterred by seeing others punished, they likely would never have been sent to prison in the first place.

Given the limited deterrent effect of punishing violent inmates, it is important to better understand potential causes and contributions to prison violence. Ideally, by having a better understanding of these factors, prison violence could be predicted with greater accuracy and programs to reduce violent acts could be implemented. For example, safeguards could immediately be put into place to reduce the likelihood of violence such as increasing staff monitoring of the high-risk inmate and decreasing his or her opportunities for violence (e.g., placing the inmate in a single cell rather than an open housing unit).

There are many environmental/situational variables that have been studied in relation to prison violence such as the security level of the prison, staff-to-inmate ratio, staff characteristics, overcrowding, and educational programming offered (Gadon, Johnstone, \& Cooke, 2006). Individual characteristics of inmates such as age, race, conviction crime, and sentence length also have been studied for their ability to predict prison violence (reviewed by Schenk \& Fremouw, 2012). An act of prison violence is generally conceptualized as an interaction of situational and 
individual-level variables. Situational variables were beyond the scope of this research study, and the primary focus was on the ability of psychological variables to predict prison violence.

The following sections summarize current research on the most replicated and consistent individual variables that were found to be predictive of prison violence, divided by demographic and criminal variables. Please refer to Schenk and Fremouw (2012) for a more detailed review and critique of other demographic and criminal variables that were not relevant to this current study. The literature surrounding the predictive utility of psychological variables is less consistent and more methodologically flawed than the literature on demographic and criminal variables, but was reviewed as justification for this current study. It also was reviewed to highlight the need for more research on these psychological variables to be able to better assess and predict risk for prison violence.

Mental health professionals are frequently called upon to assess one's risk for violence in the community. Given the volatile and potentially violent nature of prisons, it also is necessary to identify inmates' risk for violence while in prison. A better understanding of what variables to assess is needed in order for mental health professionals to more accurately identify those inmates likely to be violent. For purposes of this summary, the inmates were referred to as male because most research is conducted in male prisons and $93 \%$ of all individuals incarcerated in the United States are male (Motivans, 2013).

\section{Demographic Variables}

Age. Perhaps the most well replicated finding associated with predicting prison violence is age. Research has consistently found that as an inmate's age increases, the likelihood of engaging in violent behavior in prison decreases (e.g., Baskin, Sommers, \& Steadman, 1991; Cunningham \& Sorensen, 2006a; Cunningham \& Sorensen, 2006b; Cunningham \& Sorensen, 
2007; Cunningham, Sorensen, Vigen, \& Woods, 2010; DeLisi, 2003; DeLisi, Berg, \& Hochstetler, 2004; Edens, Poythress, Lilienfeld, Patrick, \& Test, 2008; Gillespie, 2005; Griffin \& Hepburn, 2006; Harer \& Steffensmeier, 1996; Lahm, 2008; Sorensen \& Cunningham, 2008). Based on the replication of this finding, it can be concluded that younger inmates tend to be more violent in prison than older inmates.

Race. When race is dichotomized between White and Black inmates, research indicates Black inmates are twice as likely as White inmates to have documented incidents of prison violence (Harer \& Steffensmeier, 1996). It is possible that the violent acts perpetrated by White inmates went unnoticed or undocumented by staff as a form of prejudice or bias. However, this likelihood is reduced given these results were obtained from a sample of 58 diverse federal prisons across the country. In a similar study, when inmates were dichotomized into White or non-White groups, non-White inmates also were found to be involved in more frequent and serious acts of violence in prison (DeLisi, 2003; DeLisi, Berg, \& Hochstetler, 2004).

When race is not dichotomized and is treated as a categorical variable (e.g., White, Black, Hispanic, Native American), Hispanic inmates were most likely to be violent while incarcerated in one Arizona-based study (Berg \& DeLisi, 2006). Conflicting with previous findings, one study found White inmates in another Arizona state prison were more likely to be violent than Black and Mexican American inmates (Griffin \& Hepburn, 2006).

Research findings have been fairly consistent regarding the association between race and prison violence when the variable of race is dichotomized, but this relation is less clear and consistent when multiple racial categories are examined. Geographic location of the prison can impact the racial composition of inmates and staff members and limit the generalizability of 
findings of racial differences. Given the homogenous racial composition in West Virginia, inmates in this study were dichotomized into categories of White or non-White inmates.

Education. Although the amount of schooling an inmate has completed is rarely the primary focus when attempting to predict prison violence, it has been included as a variable in some research studies. Consistently, it has been shown that the more education an inmate has received, the less likely he is to be violent while incarcerated (Berg \& DeLisi, 2006; Cunningham, Sorensen, \& Reidy, 2005; DeLisi, Berg, \& Hochstetler, 2004).

Despite these consistent findings, years of education is not necessarily directly related to intelligence level. For example, many people with low intelligence have graduated from high school due to being inappropriately passed to the next grade prior to being academically ready, while many more intelligent people have dropped out of school for various reasons. This variable could be strengthened by using objective, standardized assessment tools. In one such study, Diamond, Morris, and Barnes (2012) used the Wechsler Adult Intelligence Scale-Revised (WAIS-R) and found a negative relationship between prison violence and IQ. Specifically, as IQ scores decreased, participation in prison violence increased. The association between standardized IQ scores and prison violence is a matter that needs more examination to be better understood.

Military Experience. As of 1998, it was estimated that 56,500 Vietnam War veterans and an additional 18,500 Persian Gulf War veterans were incarcerated in state and federal prisons in the United States (Mumola, 2000). At the end of 2004, there were approximately 24,523,300 veterans residing in the United States (Noonan \& Mumola, 2007). Of this population, it was estimated that approximately 140,000 veterans were incarcerated in state or federal prisons. Veterans were incarcerated at a rate of 630 per 100,000 inmates by the end of 2004 
(Noonan \& Mumola, 2007). Macmanus and Wessely (2012) reported that military veterans in both the United States and the United Kingdom were less likely to commit a crime when compared to the overall population of these countries, but those veterans that were incarcerated were more likely to be there for committing violent and/or sexual crimes. To date, the military history of inmates has not been studied in relation to engagement in violence while in prison, but this could be a relevant relationship worthy of investigation given the high number of veterans being incarcerated for violent crimes.

\section{Criminal Variables}

Conviction Offense. In $2011,53.0 \%$ of state inmates and $9.0 \%$ of federal inmates were convicted of violent crimes (Carson \& Golinelli, 2013; Motivans, 2013). If an inmate was violent in the community leading to his incarceration, would he continue to engage in violent behaviors while in prison? Research does not support this theory of continued violence. Surprisingly, inmates convicted of violent crimes were less violent in prison than those inmates convicted of non-violent crimes (Cunningham \& Sorensen, 2007). More specifically, Cunningham, Sorensen, and Reidy (2005) found inmates convicted of property crimes were 1.5 times more likely to be violent than convicted murderers, sex offenders, robbers, and drug offenders. Similar results indicated that inmates convicted of non-violent crimes (e.g., property, drug, public order/weapon charges) were more likely to be violent in prison than inmates convicted of homicide (Sorensen \& Cunningham, 2008). Homicide offenders were $12 \%$ less likely to be violent than other violent and non-violent offenders (Cunningham \& Sorensen, 2006a). One contrasting study found inmates convicted of violent offenses were more likely to be found guilty of violent prison misconduct than inmates convicted of non-violent crimes (Griffin \& Hepburn, 2006). This was the only study that reported these conflicting results, and although statistically significant, little 
variance was actually accounted for by this relation in their model $(0.25 \% ; 0.49 \%)$. It is possible that methodological differences could have influenced these results; however, more research on the matter is necessary to be certain.

Sentence Length. It could be reasoned that inmates with long or life sentences would be more violent while incarcerated because they have less to lose. When an inmate is already serving one or multiple life sentences, additional prison time for violent acts may no longer be a deterrent. However, research results are counterintuitive to this assumption. For example, Cunningham and Sorensen (2006a) found inmates sentenced to 10-14 year and 15-19 year sentences were more likely to engage in violence than inmates serving sentences of life without parole. Inmates with long sentences (30 years or more) that were eligible for parole were the least violent group while in prison among this sample. Similarly, inmates serving a sentence between 1-10 years were more likely to be violent than inmates serving sentences of 11 years or more (Cunningham \& Sorensen, 2007). Additionally, among this sample, for each year added to an inmate's sentence, the likelihood of engaging in violence was reduced by $1 \%$ (Sorensen \& Cunningham, 2008). Research has consistently supported findings that inmates with shorter sentences tend to be more violent while incarcerated than those serving long or life sentences.

\section{Psychological Variables}

As previously noted, the research on the utility of psychological variables to predict prison violence has been scarce, as well as somewhat inconsistent or methodologically unsound. However, this relation between psychological variables and prison violence needs to be better understood due to the high rates of inmates incarcerated who have mental health needs. It was estimated in 2005 that $56 \%$ of all state inmates $(705,600), 45 \%$ of federal inmates $(78,800)$, and $64 \%$ of inmates held in jails $(479,900)$ throughout the United States had mental health problems 
(James \& Glaze, 2006). Mental health professionals are frequently called upon in correctional environments to conduct risk assessments of inmates to inform decisions, from a mental health perspective, on issues such as housing environment, security level, and frequency of mental health contacts. Empirically-supported data on the relation between psychological variables and prison violence are needed to increase the accuracy of these assessments, and ultimately, reduce and prevent occurrences of prison violence.

Major Mental Illness. With such high rates of individuals with mental health needs being incarcerated, the relation between prison violence and mental illness warrants attention. Walters (2011) examined the variables of major mental illness and general criminal thinking scores, measured by the Psychological Inventory of Criminal Thinking Styles (PICTS), as they related to violence in a federal prison. The variable of mental illness was dichotomized as "history of serious mental health problems in the form of schizophrenia or other psychotic disorder, major depression, or bipolar disorder" or no history of mental illness (p. 191). He found having a history of major mental illness accounted for a significant amount of variance when predicting violence in prison. Additionally, the general criminal thinking score from the PICTS was predictive of three or more violent acts for both mentally ill and non-mentally ill inmates. Unfortunately, measures of criminal thinking are not routinely administered to inmates at Huttonsville Correctional Center (HCC), but information on an inmate's history of major mental illness is collected upon intake for all inmates.

In a subsequent study by Walters and Crawford (2013), major mental illness was a significant predictor of violent prison behavior among 2,627 inmates. In this study, major mental illness was defined as "a reported diagnosis of schizophrenia, bipolar disorder, major depression, or serious anxiety disorder (i.e., agoraphobia, panic disorder, obsessive-compulsive disorder, and 
posttraumatic stress disorder) in the mental health section of the presentence investigation report” (p. 3, Walters \& Crawford, 2013). In this study, major mental illness and having prior convictions for violent crimes increased the probability of engaging in violent prison behavior by $28 \%$ when controlling for other variables (e.g., age, prior convictions for violence alone).

When examining perpetration and victimization of violence, Toch and Kupers (2007) reported inmates with mental health needs were disproportionately involved in violent prison incidents more than inmates without mental health needs. It was speculated this involvement could be because of their symptoms, the deinstitutionalization of mental health facilities (which led to many mentally ill people being incarcerated rather than hospitalized), and the conditions of prison, among other contributing factors. The unequal number of mentally ill inmates involved in prison violence also was found by Blitz, Wolff, and Shi (2008). When focusing on physical victimization, mentally ill inmates (based on their report of receiving mental health treatment while incarcerated) were 1.6 times more likely to be physically victimized by another inmate and 1.3 times more likely to be victimized by an employee. Research seems to suggest that mentally ill inmates are more often involved in violence, as perpetrator and victim, while incarcerated.

Aggression. Physical aggression was studied by Gillespie (2005) for the connection with self-reported involvement in prison violence. Aggression was measured based on self-report of four of the nine items from "Buss and Perry's (1992) Aggression Questionnaire" (p. 172, Gillespie, 2005). No justification was provided for why only four of the nine items of this selfreport measure were used. Regardless, it was found that as aggression scores increased, selfreported levels of prison violence also increased. Another research study similarly found selfreported inmate aggression scores were a robust predictor for both inmate-on-inmate (Lahm, 2008) and inmate-on-staff assaults (Lahm, 2009). This study also used Buss and Perry's 
Aggression Questionnaire, but only included one item from each of the four subscales of physical aggression, verbal aggression, anger, and hostility. "Space constraints of this survey" was given as a justification for only including one item from each subscale of the measure (p. 129, Lahm, 2008; p. 140, Lahm, 2009).

Walters, Duncan, and Geyer (2003) examined results from the Personality Assessment Inventory (PAI; Morey, 2007) to explore how these scales related to prison violence. The Aggression scale was initially significantly associated with involvement in prison violence; however, this finding was no longer significant after applying Bonferroni adjustments. Bonferroni corrections were appropriately applied to control Type I error because of the large number of PAI scale comparisons. Although these results did not reach statistical significance, they offered trend-level support for a connection between the personality trait of aggression and prison violence. More research is necessary to clarify the relationship between measures of aggression and prison violence.

Confusion, Depression, and Manifest Symptomology. One study examined the psychological symptoms of confusion (i.e., difficulty remembering), depression, and manifest symptomology (i.e., psychotic thought disorder) in relation to prison violence (Baskin, Sommers, \& Steadman, 1991). Scores for these symptom categories were rated based on five-items scored "never/rarely" to "always" by case managers. When controlling for other variables (e.g., age, race), confusion (i.e., memory impairment) was the only symptom related to prison violence towards other inmates and staff. As confusion ratings for an inmate increased so did his likelihood for violence.

Substance Abuse. To date, there is limited research on the relation between substance abuse and prison violence. This is an area in need of more research considering the number of 
individuals incarcerated for drug offenses or with substance abuse addictions. In 2010, most arrests (1,638,846 arrests) were for drug-related violations (e.g., manufacturing, possession, trafficking; Unified Crime Report, 2010). It is estimated that at the end of 2011, 16.8\% of all inmates $(n=225,242)$ in state prisons were incarcerated for drug offenses (Carson \& Golinelli, 2013). This proportion is much higher in federal prisons, as drug offenders made up $52 \%$ of the inmate population across federal institutions ( $n=25,416$; Motivans, 2013). Not only are many people incarcerated for drug-related crimes, but numerous inmates who are incarcerated have a history of substance abuse or current addictions. The National Center on Addiction and Substance Abuse (2010) estimated that 65\% of all inmates in the United States met the DSM-IVTR criteria for substance abuse or dependence, while another 458,000 inmates had a significant history of substance use, were under the influence at the time of their crime, committed their crime for drugs (or money to buy drugs), were incarcerated for a substance-related law violation, or some combination of these characteristics. Taken together, this amounts to $85 \%$ of the prison population in the United States having a significant substance abuse problem.

Given this frequency, research is beginning to recognize the need to include substancerelated variables when examining prison violence. Friedmann and colleagues (2008) found that violent inmates with a history of substance abuse were more likely to receive disciplinary consequences for possession of controlled substances or contraband, be diagnosed with antisocial personality disorder, or have a history of the psychotic symptom of thought insertion/control than non-violent inmates with a history of substance abuse. Specifically, inmates in this study with a history of substance abuse who received infractions for "possession or use of controlled substance" were 4.9 times more likely to engage in violent or disruptive behavior in prison than inmates with a history of substance abuse that did not receive this type of disciplinary infraction 
(Friedmann, Melnick, Jiang, \& Hamilton, 2008). This study did not include any inmates without a substance abuse history so we are unable to determine if the predictors for prison violence are different for non-substance abusing inmates.

It has been postulated that inmates with mental illness and substance use disorders will have a harder time adjusting to and coping with the demands of incarceration (Wood, 2012). To investigate this relationship further, Wood studied self-report data from 2,973 inmates currently incarcerated in federal facilities throughout the United States. To be "dually diagnosed" in this study, inmates had to have a history of mental illness and meet DSM-IV-TR criteria for substance abuse or dependence. Results of logistic regression models found inmates with a dual diagnosis were equally likely to perpetrate violent acts as non-dually diagnosed inmates; however, the dually diagnosed inmates were 1.03 times more likely to be the victim of assaults than non-dually disorder inmates. Results for inmates diagnosed with only substance-related disorders were not reported.

A similar study of 12,504 state inmates in the United States was conducted (Wood \& Buttaro, 2013). Using the same criteria for dual diagnosis (mental illness and substance use disorder), it was reported that inmates with dual diagnoses were more likely to act violently in prison than non-dually diagnosed inmates. The inmates with only a substance abuse diagnosis were less likely to be violent than the dually diagnosed inmates in this study (Wood \& Buttaro, 2013).

A noteworthy limitation of both of these studies (Wood, 2012; Wood \& Buttaro, 2013) is the reliance on self-report data for being a victim and perpetrator of prison violence. There is a need to better understand the role of substance abuse history in predicting prison violence because of the overwhelming proportion of inmates being incarcerated with addictions. 
Intelligence Quotient. As previously described, research has consistently found a negative relationship between years of education and prison violence. Educational attainment is not always directly correlated with intelligence level. Very little research has investigated the relation between IQ and prison violence using a standardized measure of intelligence. As previously mentioned when reviewing the demographic variable of education, the only such study investigating IQ and prison violence was conducted by Diamond, Morris, and Barnes in 2012. Using the WAIS-R, these researchers found that as IQ increased, engagement in prison violence decreased. Additional research utilizing an objective measure of intelligence and understanding its utility for predicting prison violence is necessary.

Despite relatively consistent findings for demographic and criminal variables, there is a great deal of inconsistency and variability found for psychological variables and their ability to predict prison violence. From which variables are studied to what measures are used to examine the determined variables, there is little agreement between researchers. Additionally, much of the research is conducted by the same researchers (e.g., Cunningham, DeLisi, Sorensen) and uses the same inmate populations (e.g., Arizona, Florida, Texas). As a result, the literature would benefit from replicating and expanding upon research of psychological variables based on standardized assessment measures to better understand the ability of these variables to predict prison violence in a novel sample.

\section{Current Study}

In the state of West Virginia, there are 14 correctional complexes ranging in security levels from minimum (an institution without a fence containing inmates with minimal security and mental health needs) to maximum security (housing the most dangerous inmates, inmates with the most severe mental health needs, or parole ineligible inmates in the state). Data from 
this study was obtained from Huttonsville Correctional Center (HCC), a medium-security institution in Huttonsville, West Virginia. HCC can house 1,138 inmates of all security levels. The majority of housing units are dormitory-style rooms with bunks or pods with four-man cells. There also are segregation units of single-celled rooms for inmates who violated institutional rules, are new to the facility, or are in protective custody. Again, situational variables were not examined as data were only collected from one prison in the state of West Virginia. Upon intake to HCC, inmates are administered a standard battery of assessments (e.g., Minnesota Multiphasic Personality Inventory-2-Restructured Form, Beta-III, and Texas Christian University Drug Screener II) and a psychological evaluation is completed to assess mental health history and current mental health needs. Data were collected from files of this population of inmates to investigate the following three purposes.

\section{The primary purpose of this study was to determine if psychological measures} add to the prediction of violence in prison.

Previous research has replicated findings of demographic and criminal variables being consistently predictive of identifying those inmates who engage in prison violence from those who do not. In addition to these variables, different psychological constructs have been studied related to prison violence. To date, the results of these studies examining psychological variables have been inconsistently replicated, have not yet been replicated at all, or have significant methodological flaws making the relation between these variables and prison violence unclear.

Given the variability in the findings of previous research, the primary purpose of this study was to clarify the predictive ability of psychological variables to determine the violent from non-violent inmates. The psychological variables studied included: history of mental illness, personality characteristics and psychopathologies (MMPI-2-RF; Ben-Porath \& Tellegen, 
2008), intelligence (Beta-III; Kellogg \& Morton, 1999), and substance abuse (TCU Drug Screen II; Knight, Simpson, \& Morey, 2002).

\section{Purpose 1 Hypotheses}

1.1. Research on the relation between mental illness and perpetration of prison violence has found inmates with a history of mental illness tend to engage in more violent prison behavior than inmates without a history of mental illness (Toch \& Kupers, 2007; Walters, 2011; Walters \& Crawford, 2013). Based on these findings, it was hypothesized that inmates in this study with a history of mental illness would be more likely to be violent than inmates without a history of mental illness.

1.2. Previous research has found self-reported aggression to be predictive of prison violence (Gillespie, 2005; Lahm, 2008; Lahm, 2009; Walters, Duncan, \& Geyer, 2003). As a result of these findings, it was hypothesized that inmates who endorsed more items on a scale of Aggression (e.g., Aggression, Aggressiveness-Revised, Anger Proneness, Antisocial Behaviors, Behavioral Externalization), as measured on the MMPI-2-RF, would engage in more violence while incarcerated.

1.3. Baskin, Sommers, and Steadman (1991) investigated different symptomology of mental illnesses and found that symptoms of confusion, particularly memory impairments, were predictive of violent behavior among inmates when controlling for other demographic and criminal history variables. The Cognitive Complaints scale on the MMPI-2-RF encompasses similar symptoms of memory impairment (Ben-Porath, 2012). Considering the results of previous research, it was hypothesized that inmates who had higher scores on the Cognitive Complaints scale of the MMPI-2-RF (which results from endorsing more memory/concentration 
problems) would be more likely to engage in prison violence than inmates with lower scores on this scale.

1.4. Research has consistently found that the more education an inmate has received in the community, the less likely he is to be involved in violent acts while incarcerated (Berg and DeLisi, 2006; Cunningham, Sorensen, \& Reidy, 2005; DeLisi, Berg, \& Hochstetler, 2004). However, there are inherent flaws associated with the variable of years of education completed that do not automatically align with intelligence levels. Therefore, scores on an intelligence assessment measure (Beta-III) were examined. Consistent with a previous study (Diamond, Morris, \& Barnes, 2012), it was hypothesized that individuals with lower Beta-III intelligence scores would engage in more prison violence than those inmates with higher Beta-III IQ scores.

1.5. To date, there has been very little research examining the relation between substance abuse and prison violence. In the research that has been conducted, inmates with substance abuse disorders were more likely to be violent when they have a history of thought insertion/control, a diagnosis of antisocial personality disorder, and receive disciplinary infractions for possession of a controlled substance, but we do not know how this differs from inmates without substance abuse problems (Friedmann et al., 2008). Other research has found inmates meeting diagnostic criteria for substance abuse or dependence were less likely to be violent in prison than inmates with both a substance abuse/dependence diagnosis and severe mental illness (Wood \& Buttaro, 2013); however, we still do not know if inmates with a substance abuse history will be more or less violent than inmates without a substance abuse history. The current study included the variable of scores on the Texas Christian University Drug Screen II (TCU Drug Screen II; Knight, Simpson, \& Morey, 2002), a measure of substance dependence, to determine if selfreported substance dependence adds any predictive utility in identifying who will be violent in 
prison from who will not be violent. Because of the mixed results of previous research and the novel nature of using the TCU Drug Screen II in this capacity, no specific hypotheses were made regarding the predictive ability, if any, between self-reported substance dependence and prison violence.

\section{The second purpose of this study was to devise a weighted score to reflect} different degrees of severity of violent acts that occur in prison and use this score as a continuous dependent variable to better understand the ability of psychological variables to predict the severity of violence.

The same demographic (age, race) and criminal variables (crime, sentence length) were included to better understand if psychological variables could predict severity of violence over and above these other categories. This purpose was exploratory in nature and hypotheses were consistent with Purpose 1.

To create this Violence Severity Index score (VSI), the incident reports for violent acts were given a score of $0=$ none (no documented violence), $1=$ mild violence (one victim, no weapon), 2 = moderate violence (use of a weapon and/or injury requiring first-aid procedures), and $3=$ severe violence (more than first aid medical attention required). Inmates with multiple incident reports for violent acts had the score of each act summed to get a total gauge of the overall severity. For example, an inmate has four violent incident reports: two were in the mild category and two were moderately violent. His VSI score would be six $(1+1+2+2)$. Similarly, an inmate with eight separate mild acts of prison violence would have an overall score of eight $(1+1+1+1+1+1+1+1)$. An inmate who has no violent incident reports would have a VSI score of zero. This method to create a weighted Violence Severity Index is similar to the Cormier-Lang System for Quantifying Criminal History used in risk prediction and recidivism research 
(Quinsey, Harris, Rice, \& Cormier, 2006). This rating of violent prison behavior is novel; however, if demographic, criminal, or psychological variables are able to accurately predict serious prison violence, risk can be assessed more succinctly and steps can be taken to prevent these significant and damaging acts of prison violence.

\section{The third purpose of this study was to determine if the personality psychopathology scales on the MMPI-2-RF could form meaningful clusters of violent inmates.}

To date, no research has attempted to identify clusters, or similar groupings of inmate characteristics, of those who engage in prison violence. For example, do some inmates engage in violence primarily prompted by severe mental illness while other violent inmates are void of mental illness but are impulsive and aggressive in disposition? The five personality psychopathology scales on the MMPI-2-RF include: Aggressiveness, Psychoticism, Disconstraint, Negative Emotionality/Neuroticism, and Introversion/Low Positive Emotionality. Because the MMPI-2-RF is relatively new to being used and researched in correctional settings, and because clusters of violent inmates have not been identified yet, it would be helpful to understand if any of these five simple scales of personality psychopathology could group violent inmates based on commonalities in score patterns. This finding could contribute to a systematic, brief, and ideally accurate assessment to identify which types of inmates would be more likely to be violent while incarcerated. Although a certain score on one of these five scales could not independently predict which inmates would be violent, scores on these personality scales could combine with other identified demographic, criminal, and psychological information to enhance the understanding of violent inmates and more accurately identify these inmates who are at risk for violence in prison.

\section{Purpose 3 Hypotheses}


The purpose of determining if clusters of violent inmates emerge using the five personality psychopathology scales on the MMPI-2-RF was exploratory in nature. However, based on previous findings that inmates who endorse more aggressive tendencies are more violent in prison (e.g., Gillespie, 2005; Lahm, 2008; Lahm, 2009; Walters, Duncan, \& Geyer, 2003), it was expected that a cluster of violent inmates would have higher Aggression and/or Disconstraint (e.g., impulsivity, acting out) scale scores than other clusters. Another group that was thought to emerge based on these five scales was based on the Psychoticism scale. People suffering from delusions and hallucinations are not oriented to reality and have disorganized behaviors. They become more impulsive and unpredictable which could increase their likelihood of violence. Psychoticism also is the only one of these five scales that captures major mental illness which previous research has shown is often associated with increased violent behavior in prison (Toch \& Kupers, 2007; Walters, 2011; Walters \& Crawford, 2013). It was speculated that no clusters of violent inmates would be grouped by Negative Emotionality/Neuroticism or Introversion/Low Positive Emotionality, as these presentations are indicative of more withdrawn, self-containing actions rather than extroverted, acting-out displays of behavior.

\section{Method}

\section{Participants}

The inmates at Huttonsville Correctional Center (HCC) are all male, over the age 18, and have been convicted of committing a crime in the state of West Virginia. More specifically, individuals convicted of any felony crime and sentenced to any imprisonment length, except Life without Mercy, are housed at HCC. Based upon behaviors while incarcerated, inmates can be transferred to more or less secure correctional institutions within the state. For example, if an inmate perpetrates a serious violent act at HCC, they could be transferred to the state's 
maximum-security prison. Similarly, inmates that are nearing the end of their sentence and display stable, appropriate conduct could be transferred to a less secure institution. As a result of this constant fluidity among the inmate population, it is estimated that $30 \%$ of the inmate population at HCC turns over every six months (T. Horacek, personal communication, October 19, 2012). Data were collected multiple times over the course of three months, which allowed for a larger, more generalizable and representative sample for this study. Situational variables were not analyzed as data were only collected from one specific institution.

Inmates that displayed physically aggressive behaviors while in prison were classified by staff as "Assaultive" and their records were labeled to reflect this risk. In addition to this label, there was documentation kept within the file that described the violent act to substantiate the reason for this classification. Other risk classification categories an inmate could receive based on their actions while in prison were: Threatening, Threatened, Suicide Risk, Escape Risk, Registrant (i.e., instant offense was a sexual crime), Sexual Predator (i.e., perpetrated sexual acts while incarcerated), and Separation Issues (i.e., if the inmate had a problem with another (or other) inmate(s) and needs to be physically separated in order to prevent violence). Inmates could have as many of these risk classifications as warranted. This "flagging" system was a way to communicate particular risk factors of an inmate to staff.

As previously explained, there are downfalls for relying upon official documentation of violent behavior. This type of data is subject to underreporting (e.g., Bryne \& Hummer, 2007) and biased reporting. After considering the limitations of relying on official documentation of violence, this method was still determined to be more reliable than inmate self-report. The nature of prisons makes it unlikely to produce accurate estimates of violence, as the culture discourages reporting misbehavior of inmates by other inmates ("snitching"). If given the opportunity to 
anonymously self-disclose prison violence, there is no guarantee that inmates would be accurate or honest in their estimates, as their credibility has been tarnished from their incarcerationworthy behaviors. Research also indicates biases in self-disclosure of prison violence based merely on how the question is phrased (Wolff, Shi, \& Bachman, 2008). Also, inmates might be more likely to self-report being violent in hopes of appearing "tough" or "macho" than selfreport victimization in order to not appear weak. Just the opposite might be true for other inmates, who would be more likely to report victimization than perpetration of violence to avoid getting in trouble for admitting violent behavior. There are a multitude of ways self-report data can be manipulated based upon the cultural influences of the prison environment. Weighing the benefits and drawbacks to both types of data collection, it was determined that utilizing official documentation would likely yield a more conservative, but accurate estimate of prison violence.

For the purpose of this study, the records of all inmates at HCC with an "Assaultive" risk classification were reviewed. These inmates were deemed the most dangerous and violent by staff based on having one serious incident of violence while incarcerated or perpetrating numerous acts of more minor violence while incarcerated. Throughout the three month data collection period, there were 60 inmates designated as "Assaultive," which comprised the violent sample of this study. To ensure this sample included inmates that were physically violent while incarcerated, documentation explaining the critical incident that resulted in the "Assaultive" risk classification was reviewed. Incidents of only verbal aggression, threats, posturing, possession (without use) of a weapon, and violence towards oneself would have been excluded from review. However, all inmates marked as "Assaultive" were found to have perpetrated acts of physical violence towards others so no files had to be excluded from being reviewed based on this criterion. 
Of the 60 violent inmates, $30(50.0 \%)$ assaulted other inmates and $16(26.7 \%)$ assaulted staff. An additional ten inmates $(16.7 \%)$ had both inmate and staff victims of their violence. There was limited documentation for four inmates, making it impossible to ascertain who the victim of their violence was. Although there were only 60 inmates designated as "Assaultive," the violent acts they engaged in were typically very severe. For example, one staff member was beaten with a two-pound weight and suffered a skull fracture. Another inmate kicked and punched a staff member in the head, which required facial reconstruction surgery. There also was an incident of an inmate tying up another inmate, tattooing him against his will, and assaulting him over an extended period of time. These extreme acts of violence resulted in different punishments. One common punishment was being sentenced to a solitary confinement unit for a period of time ranging anywhere from seven days to two years; however, the normative sentence for solitary confinement was 60 days.

Because of the relatively small number of violent inmates (60 inmates out of 1,138 , $5.3 \%$ ), a random sample of twice as many inmates was selected from the remaining population to comprise the "control"1 group $(n=120)$. This sampling was done randomly using a roster of inmates numbered 1 through 1,078 with the names of violent inmates removed. Numbers were chosen using a random number generator. Inmates were not matched by age, race, or other demographic characteristics as these variables were entered into the regression model to determine if they accounted for a significant amount of variance.

\section{Materials}

\footnotetext{
${ }^{1}$ The term control group was used to represent the sample of 120 inmates randomly selected from the population of inmates at HCC. Although the term "control" typically indicates a group not being experimentally manipulated, referring to this group by another term would likely be more cumbersome (e.g., general population inmates, inmates not flagged as assaultive) or deceiving (e.g., non-violent group, which would be inaccurate as some of the inmates randomly chosen have engaged in violence but not to the severity to warrant the "Assaultive" risk designation).
} 
At Huttonsville Correctional Center (HCC), all inmates completed the MMPI-2-RF (BenPorath \& Tellegen, 2008), Beta-III (Kellogg \& Morton, 1999), and TCU Drug Screen II (Knight, Simpson, \& Morey, 2002) upon intake to the facility. Psychology staff also completed a psychological evaluation of inmates, which included a mental health history and current mental health and status assessment. The results of these assessments and the evaluation were kept in each inmate's file and records were stored in a secure office that was only accessible to authorized employees.

Demographic and Criminal Variables. The following demographic information was collected from the records of selected inmates: age (at intake and time of violent act, if applicable), race, years of education, military history, and military discharge, if applicable. This information was collected from the West Virginia Division of Corrections Parole Services Investigation Report found in every inmate record.

Additionally, the crime the inmate was convicted of and his sentence was recorded. Type of crime was separated into violent, sexual, property, drug, and "other" (e.g., public order/weapons crimes) categories, which are similar to categories used by Cunningham and Sorensen (2006a) with the exception of distinguishing violent crimes from sexual crime. This distinction was made to explore if there are differences in predicting prison violence between these types of offenders who have qualitatively different crimes. Another variation is this study did not have a distinct homicide group due to the limited number of these offenders at HCC. Previous studies had a much larger population, which allowed for this distinct group to be analyzed (e.g., Cunningham \& Sorensen, 2006a). Inmates convicted of multiple crimes were categorized by the most severe, ranging from violent, sexual, property, drug, and "other" (e.g., public order/weapon) offenses. 
Sentence length was recorded for each inmate. In the case of multiple sentences, how sentences were run (concurrently or consecutively) was taken into consideration when documented. The inmate's parole eligibility date also was noted in order to calculate the minimum sentence the inmate would serve before becoming parole eligible. This crime-related information was collected from the West Virginia Court Order documentation within each record.

Mental Health History. As previously noted, a psychological evaluation is completed shortly after an inmate's arrival to the facility. If the inmate is transferred from another facility in the state, a psychological evaluation should already be in the inmate's file. This evaluation includes information on the inmate's current mental health needs and mental status, as well as any available information on the inmate's history of mental illness. For the current study, mental health history was dichotomized into a yes/no response based on information contained in this report. An inmate was determined to have a history of mental health needs ("yes") if they were ever diagnosed with a psychological disorder or received mental health treatment of any kind (i.e., psychopharmacological, therapy, hospitalization). An inmate was determined to not have a history of mental health needs ("no") if there was no history of a diagnosis for a psychological disorder or no history of mental health treatment.

\section{Minnesota Multiphasic Personality Inventory-2-Restructured Form (MMPI-2-RF).}

The MMPI-2-RF is a shortened version of the Minnesota Multiphasic Personality Inventory-2 (MMPI-2) to assess psychopathology. It is derived directly from the 567-items from the MMPI2. The Minnesota Multiphasic Personality Inventory-2 (MMPI-2; Ben-Porath \& Tellegen, 2008) was a revision of the original MMPI, and is now one of the most widely used, researched, and accepted measures of adult personality characteristics and psychopathologies in the United States 
(Graham, 2011). Using the MMPI-2 profiles, research has identified classification rules (i.e., Megargee offender classification system) for male, female, state, and federal inmates throughout the country (Megagree, 1994; 1997; Megargee, Mercer, \& Carbonell, 1999). However, the MMPI-2 has yet to demonstrate the ability to predict inmates that will be violent while incarcerated with much certainty (Cunningham \& Sorensen, 2006b). To date, there has been no research conducted on the utility of the newer MMPI-2-RF to predict violence in prison or to classify different categories of inmates.

The MMPI-2-RF is 338-item, self-report questionnaire that measures different components of personality and psychopathology (Ben-Porath \& Tellegen, 2008). These 338items are directly from the 567-items on the MMPI-2. All questions on the MMPI-2-RF are answered "True" or "False." This assessment tool has a Flesch-Kincaid reading level of grade 4.5 and is appropriate to administer to individuals 18 years old and older. Additionally, individual results can be compared to particular reference groups, such as medical samples and correctional populations.

The MMPI-2-RF has nine validity scales (variable response inconsistency, true response inconsistency, infrequent responses, infrequent psychopathology response, infrequent somatic responses, symptom validity, response bias scale, uncommon virtues, and adjustment validity), three Higher-Order scales (emotional/internalizing dysfunction, thought dysfunction, behavioral/externalizing dysfunction), and nine Restructured Clinical scales (demoralization, somatic complaints, low positive emotions, cynicism, antisocial behavior, ideas of persecution, dysfunctional negative emotions, aberrant experiences, hypomanic activation). There also are five Somatic scales (malaise, gastrointestinal complaints, head pain complaints, neurological complaints, cognitive complaints), nine Internalizing scales (suicidal/death ideation, 
helplessness/hopelessness, self-doubt, inefficacy, stress/worry, anxiety, anger proneness, behavior-restricting fears, multiple specific fears), four Externalizing scales (juvenile conduct problems, substance abuse, aggression, activation), five Interpersonal scales (family problems, interpersonal passivity, social avoidance, shyness, disaffiliativeness), and two Interest scales (aesthetic-literary interests, mechanical-physical interests). Lastly, the MMPI-2-RF profile contains five Personality Psychopathology scales (aggressiveness-revised, psychoticismrevised, disconstraint-revised, negative emotionality/neuroticism-revised, introversion/low positive emotionality-revised).

The MMPI-2-RF was normed based on 2,276 individuals across the United States, ranging in age from 18-80 years old (Ben-Porath \& Tellegen, 2008). As a result of the items on the MMPI-2-RF being directly derived from the MMPI-2, the MMPI-2-RF was validated on preexisting MMPI-2 samples by the creators of the measure (Tellegen \& Ben-Porath, 2008). Van Der Heijden, Egger, and Derksen (2010) also successfully replicated the validation of the MMPI2-RF based on the MMPI-2 with another sample of international participants. In addition, the MMPI-2-RF has been validated against measures of psychopathy, fearless-dominance, and impulsive-antisociality (Sellbom, Ben-Porath, Patrick, Wygant, Gartland, \& Stafford, 2012). Many other studies have examined the psychometric properties of specific subscales on the MMPI-2-RF such as the Response Bias Scale (RBS; Wygant, Sellbom, Gervais, Ben-Porath, Stafford, Freeman \& Heilbronner, 2010) and Somatic Complaints scale (Thomas \& Locke, 2010).

Previous research has used the cut-off values specified in the interpretation manual published by test developers to identify invalid results (Marion, Sellbom, Salekin, Toomey, Kucharski, \& Duncan, 2013; Sellbom \& Bagby, 2008; Tarescavage, Wygant, Boutacoff, \& Ben- 
Porath, 2013). However, previous research has found that some invalid profiles among prison inmates can be informative for different behaviors such as sex offender completion versus dropout (Clegg, Fremouw, Horacek, Cole, \& Schwartz, 2010). Because intentionally providing false information on this assessment is still a form of information, all completed MMPI-2-RFs were included in the current analyses. It also was decided to include all completed MMPI-2-RF results because of the small sample size of the current study. Removing invalid MMPI-2-RF protocols would have decreased the sample size from 55 violent inmates who completed the measure to 45 usable protocols, and from 120 control inmates to 114 valid protocols $(n=159)^{2}$.

Beta-III Intelligence Assessment. The Beta-III is a measure of non-verbal intelligence (Kellogg \& Morton, 1999). It can be administered to individuals from 16 to 89 years old. The subtests on the Beta-III include: Coding, Picture Completion, Clerical Checking, Picture Absurdities, and Matrix Reasoning. The final score produced by the Beta-III is an intelligence quotient (IQ) that can theoretically range from 0 to $150+$, but the average IQ score is 100 (Neisser, 1997). Scores of 70 and below are considered when diagnosing an intellectual disability, as specified by the Diagnostic and Statistical Manuel, $5^{\text {th }}$ Edition (American Psychiatric Association, 2013).

The Beta-III is the second revision of the original Beta, which was initially designed for use with military personnel (Pearson Education, Inc., 2012). The Beta-III was normed on a sample of 1,260 adults and validated on samples of mentally retarded individuals and inmates (Kellogg \& Morton, 1999). Additionally this version of the intelligence assessment measure has been validated against other intelligence tests such as Wechsler Adult Intelligence Scale-III (WAIS-III) and Beta-II (as reported by Pearson Education, Inc., 2012).

\footnotetext{
${ }^{2}$ Analyses were conducted with invalid MMPI-2-RF protocols removed $(n=45)$. Psychoticism was no longer significantly different between violent and control group inmates; however, there were no other meaningful differences on the logistic regression, multiple regression, and cluster analyses.
} 


\section{Texas Christian University Drug Screen-II (TCU Drug Screen II). The TCU Drug}

Screen II is a nine-item questionnaire assessing substance use that can be given as an interview or self-report measure (Knight, Simpson, \& Morey, 2002). Each question is answered with a "yes" or "no" response and total scores are calculated by summing one point per every "yes" response (Appendix A). Total scores range from zero to nine points. A total score of three points or more is indicative of a "relatively severe drug problem" and correlates with a Diagnostic and Statistical Manual, $4^{\text {th }}$ Edition-Text Revision diagnosis for a substance dependence disorder (p. 4, Knight, Simpson, \& Morey, 2002). An additional six questions on the TCU Drug Screen II are not included in the calculation of the total score, but provide qualitative information regarding which drugs were most problematic for the respondent, frequency of use in the past year, substance abuse treatment history, and insight into how problematic one's drug use is and need for treatment.

The original TCU Drug Screen was created by the same authors for the purpose of developing an efficient and effective screening tool to assess substance use histories and make appropriate substance abuse treatment referrals within correctional settings in the United States (Knight, Simpson, \& Morey, 2002). Using a sample of 18,364 male and female inmates within the Texas Department of Criminal Justice, the TCU Drug Screen was found to have an overall Cronbach's alpha level of .89, indicating acceptable reliability of the items. Using this large sample, responses were normed by gender, race, age groups, and most serious drug problem. Test-retest reliability one week apart and validation with other substance use measures (e.g., Substance Abuse Subtle Screening Inventory-2, Drug Abuse Screening Test) has been conducted with the TCU Drug Screen (Peters et al., 2000). Test-retest reliability produced a Pearson's product-moment correlation of 0.95 and accurately identified $82.0 \%$ those with alcohol or drug 
abuse disorders $(n=328)$. The TCU Drug Screen was marginally modified based on feedback from respondents to form the TCU Drug Screen II, but the psychometric properties remained equivalent (Knight, Simpson, \& Morey, 2002).

\section{Procedures}

Permission to conduct this study was obtained from Commissioner Rubenstein, the Commissioner of Corrections in West Virginia, Warden Plumley, the Warden of Huttonsville Correctional Center, and PsiMed, Inc. Approval to conduct this research as proposed also was obtained from the Institutional Review Board (IRB) of West Virginia University.

As previously described, all inmates who were classified as an "Assaultive" risk were reviewed $(n=60)$. Due to the relatively small number of inmates carrying this designation, a sample of twice as many inmates $(n=120)$ from the remaining population were randomly selected in order to more accurately represent the characteristics of the entire inmate population at HCC. Random sampling of the remaining population was done using a random number generator and a roster of inmates incarcerated at $\mathrm{HCC}$ at the time of data collection (violent inmates' names were removed). Inmates in the control group were not matched to inmates in the violent group by any demographic characteristics because these variables were entered into the regression equations to determine how much variance they account for among other variables.

\section{Reliability Procedures}

The primary researcher coded all of the collected data (please refer to Appendix B for the data collection form used). To ensure that a researcher bias was not present, a trained graduate student recoded 44 of 180 cases (24.4\%) to verify interrater reliability and rule-out potential biases in scoring. Interrater reliability was analyzed using the Kappa statistic for agreement of dichotomous and categorical variables (e.g., military history $=$ yes or no; racial category $=$ white 
or non-white) and intra-class correlation coefficients (ICC) values were calculated for continuous variables (e.g., years of education). Interrater reliability ranged from Kappa values of 0.82 to 1.00 for dichotomous and categorical variables, and ICC values of 0.74 to 1.00 for continuous variables. Tables 1 and 2 display a complete listing of all Kappa and ICC values, respectively. Any discrepancies between the primary researcher and graduate student were discussed. The appropriate manner in which to code the disputed variable was agreed upon by both the primary researcher and graduate assistant after consulting the documentation contained in the inmate's file. All discrepancies were resolved.

\section{Results}

\section{Demographic and Univariate Analyses}

The final sample for this study included 60 violent inmates and 120 control inmates. Table 3 presents the dichotomous and categorical demographic, criminal, and psychological characteristics of the sample, as well as chi-square analysis results. Consistent with previous research (e.g., DeLisi, 2003; DeLisi, et al., 2004; Harer \& Steffensmeirer, 1996) and due to the homogeneity of racial composition in West Virginia, inmates included in the final sample for this study were divided into racial categories of white and non-white. The category of non-white inmates included inmates who were identified as Black, Hispanic, Native American, Asian, or biracial. The violent group of inmates was comprised of 41 (68.3\%) white inmates and 19 (31.7\%) non-white inmates, whereas the control group was comprised of $99(82.5 \%)$ white inmates and $21(17.5 \%)$ non-white inmates. The difference in racial composition between violent and control groups was significantly different, $\chi^{2}(1, N=180)=4.65, p=.031, \varphi=-0.16($ small effect size $)^{3}$. Seven inmates in the violent group (11.7\%) and $16(13.3 \%)$ inmates in the control group were military veterans. There was a significant difference between those receiving

\footnotetext{
${ }^{3}$ The small, medium, and large effect size approximations throughout this document are based on Nandy (2012).
} 
honorable and those receiving dishonorable discharges from the military, $\chi^{2}(2, N=180)=7.71$, $p=.021, \varphi=0.21$ (small effect size). Specifically, five of the seven violent group inmates with a military history were dishonorably discharged (71.4\%) whereas only two of the 16 control group veterans were dishonorably discharged (12.5\%).

The criminal variable of conviction offense was examined for differences between the violent and control group inmates. Conviction offenses were separated into the following categories: violent, sex, drug, property, and "other" crimes. "Other" crimes could include offenses such as public order or weapons crimes. There was a significant difference in the conviction offense between the violent and control groups, $\chi^{2}(4, N=180)=10.74, p=.030, \varphi_{c}=$ 0.24 (small effect size). The majority of inmates in both the violent and control groups were convicted of a violent offense. However, $50 \%$ of violent group inmates $(n=30)$ were convicted of a violent crime, compared to only $29.2 \%$ of inmates in the control group $(n=35)$. Control group inmates also were more likely to be convicted of drug crimes $(n=19,15.8 \%)$ than violent group inmates ( $n=5,8.3 \%$ ), as well as "other" crimes (control: $n=12,10.0 \%$, violent: $n=1$, $1.7 \%)$

In terms of psychological variables, significantly more violent inmates had a history of mental illness $(n=37,61.7 \%)$ than control group inmates $(n=51,42.5 \%), \chi^{2}(1, N=180)=$ $5.88, p=.02, \varphi=0.18$ (small effect size). Violent inmates also refused to complete the MMPI-2RF more frequently $(n=5,8.3 \%)$ than control group inmates $(n=0,0.0 \%), \chi^{2}(1, N=180)=$ $10.29, p=.001, \varphi=-0.24$ (small effect size). Of those inmates that did agree to complete this measure, significantly more MMPI-2-RF profiles were invalid for violent inmates $(n=10$, $18.2 \%)$ than non-violent inmates $(n=6,5.0 \%), \chi^{2}(1, N=180)=7.89, p=.01, \varphi=-0.22($ small effect size). 
Table 4 summarizes the descriptive statistics of continuous demographic, criminal, and psychological variables for the sample, as well as presents the results of univariate analyses for these variables. There was a significant difference between the violent and control group inmates for the variable of age of incarceration, $t(174)=-3.27, p=.01, d=0.53$ (medium effect size). Violent inmates were incarcerated at a younger age $(M=27.40, S D=9.08)$ than control group inmates $(M=32.62, S D=10.54)$. Violent inmates faced sentences ranging in length from one year to life with mercy, with the mean sentence for this group being 8.79 years $(S D=9.51)$. The mean sentence for control group inmates was 6.15 years $(S D=6.50)$, and also ranged from one year to life with mercy. A total of $18.3 \%$ of violent inmates $(n=11)$ were sentenced to life with mercy, whereas only $7.5 \%$ of control group inmates $(n=9)$ received this sentence. There was a significant difference in the sentence length for violent and control group inmates, $t(179)=2.19$, $p=.03, d=-0.32$ (small effect size).

Violence Severity Index (VSI) scores were calculated for all inmates based on violent acts they engaged in while incarcerated. These scores ranged from zero to eleven. Violent inmates had significantly higher VSI scores $(M=3.38, S D=2.15)$ than control group inmates $(M=0.16, S D=0.52), t(178)=15.60, p<.001, d=-2.06$ (large effect size). Because control group inmates were randomly selected from the population, some had engaged in violence but the majority did not $(n=108,90.0 \%)$. VSI scores for control group inmates were scores of one $(n=6,5.0 \%)$, two $(n=5,4.2 \%)$, or three $(n=1,0.8 \%)$. An interesting trend-level finding was that VSI scores significantly differed based on the victim of the violent act, $F(3,55)=2.65, p=$ 0.058; Wilks' $\lambda=0.62$, partial $\eta^{2}=0.13$ (medium effect size). Inmates who only assaulted staff members had the lowest VSI scores $(M=2.69, S D=2.50)$, whereas inmates who assaulted both 
staff and other inmates had the highest VSI scores $(M=4.70, S D=1.89)$. Inmates who only assaulted other inmates had moderated VSI scores $(M=3.50, S D=1.96)$.

Both groups completed comparable years of schooling. The two groups also did not differ on Beta-III IQ or TCU Drug Screen II scores. Specific MMPI-2-RF scales were hypothesized to be meaningful and analyzed based on previous literature (see literature review and proposed hypotheses). The violent and control group inmates did not differ on their Cognitive Complaints scale scores on the MMPI-2-RF. There are five subscales on the MMPI-2-RF that tap into the construct of aggression: Behavioral Externalization Dysfunction, Antisocial Behavior, Anger Proneness, Aggression, Aggression-Revised. A MANOVA was conducted to analyze these five scales. To account for the unequal cell sizes and some missing values on the MMPI-2-RF (inmates who refused to complete the measure), Sum of Squares Type IV was used (Shaw \& Mitchell-Olds, 1993). Also due to the unequal group sizes and the sensitivity to violations in normality, a significance value of $p=.001$ was used to evaluate violations of Box's $M$ (Tabachnick \& Fidell, 2007). With these adjustments, all assumptions were met and Box's $M$ was not significant. The MANOVA results indicated there were no overall differences between groups on these five scales, $F(5,169)=1.63, p=0.15 ;$ Wilks' $\lambda=0.95$, partial $\eta^{2}=0.05$. When examining the univariate analyses, there also were no significant differences between violent and control group inmates on any of these five scales. Table 4 displays these univariate results.

Another MANOVA was conducted to identify if any significant differences existed among the violent and control groups on the five Personality Psychopathology scales on the MMPI-2-RF, which include: Aggressiveness, Psychoticism, Disconstraint, Negative Emotionality/Neuroticism, and Introversion/Low Positive Emotionality. Consistent with the previous MANOVA, Sum of Squares IV and a significance value of $p=.001$ for Box's $M$ was 
used. With this value, Box's $M$ statistic was not significant and all assumptions for this statistical analysis were met. The overall MANOVA for the five personality scales was approaching significance, $F(5,169)=2.00, p=0.08 ;$ Wilks' $\lambda=0.94$, partial $\eta^{2}=0.06$ (medium effect size). Examination of univariate analyses indicated that the only scale of the five personality scales on the MMPI-2-RF that reached statistical significance with Bonferroni corrections was Psychoticism, $F(1,173)=8.24, p=.005$, partial $\eta^{2}=.05$ (small to medium effect size). Table 5 presents the results of the univariate analyses for the five MMPI-2-RF Personality Psychopathology scales.

The hypothesis that a history of mental illness would be more prevalent among violent inmates than control group inmates was supported. The hypotheses that violent inmates would have significantly higher scores on MMPI-2-RF scales of aggression and Cognitive Complaints were not supported. Results also did not support the hypotheses that violent inmates would have lower IQs on the Beta-III than control group inmates, and that these two groups would differ on their self-reported substance abuse histories, as measured by the TCU Drug Screen II. Nonsignificant findings were not entered into the subsequent regressions.

\section{Purpose 1—Logistic Regression Results}

A binary logistic regression was performed to assess the impact of the significant demographic, criminal, and psychological variables on predicting violent from control group inmates. The model contained six predictor variables: age and race (demographics), conviction crime and sentence length (criminal variables), and mental health history and the MMPI-2-RF scale of Psychoticism (psychological variables). Table 6 displays results of bivariate correlations between these six predictor variables entered into both logistic and multiple regressions. This model had three levels. Demographic variables were entered at the first level of the regression 
because they have been extensively replicated and supported the most consistently by previous research. The criminal variables were entered in the next step because research also supports their utility in predicting violence and they should be controlled for before the unknown psychological variables were entered in the third step. Mental health history and the MMPI-2-RF scale of Psychoticism were entered in the third and final step of the logistic regression. Because of the limited sample size and the lack of significant findings from univariate analyses, other psychological variables (e.g., aggression scales, Cognitive Complaints scale) were not included. The criterion variable was the violent and control inmate grouping.

The full model containing all predictors was statistically significant, $\chi^{2}(9, N=180)=$ 41.73, $p<.001$, indicating that the full model was able to distinguish between violent and control inmates. The model as a whole explained between $21.4 \%$ (Cox and Snell R square) and $30.0 \%$ (Nagelkerke R squared) of the variance in group status, and correctly classified $73.4 \%$ of cases.

As shown in Table 7, only three of the predictor variables made a unique, statistically significant contribution to the full model: age at incarceration, mental health history, and Psychoticism. Variables of sentence length and the conviction offense of property crimes were approaching trend level significance. The strongest predictor of identifying violent inmates from control group inmates was mental health history with an odds ratio of 2.68. An odds ratio of 2.68 indicates inmates with a mental health history were more than twice as likely to engage in violent behaviors while incarcerated, when controlling for all other factors in the model. An odds ratio of 1.03 for the MMPI-2-RF scale of Psychoticism means inmates with higher scores on this scale were more likely to be in the violent group than the control group. The odds ratio of .93 for age 
was less than one, indicating that for every additional year of age, inmates were .93 times less likely to engage in prison violence, controlling for other factors in the model.

Table 8 shows the sensitivity and specificity of the model. Specifically, the sensitivity for the model with all six predictors entered is $40.0 \%$, meaning the model was able to correctly classify $40.0 \%$ of the inmates that were violent. This is the highest sensitivity value for the three blocks of variables. The specificity for the model with all six predictor variables entered is 89.0\%. Eighty-nine percent of control group inmates were accurately predicted to be in the control group.

\section{Purpose 2-Hierarchical Multiple Regression Results}

The second purpose of this study was to conduct a hierarchical multiple regression analysis predicting Violence Severity Index (VSI) scores. The Violence Severity Index (VSI) score is a weighted calculation based upon the severity and frequency of violent acts in prison (please refer to Purpose 2 for a description of how VSI scores were calculated). Because the control group was comprised of inmates randomly selected from the remaining population of inmates that were not designated as "Assaultive," it was possible for these inmates to have engaged in violence while incarcerated, but likely not to the severity of the violent group. There were 12 inmates in the control group who had a VSI score of one or more because they engaged in violence while incarcerated. VSI Scores ranged from zero to 11, and the entire sample had a mean VSI score of $1.23(S D=2.01)$. When the two groups were examined separately, violent inmates had significantly higher VSI scores $(M=3.38, S D=2.15)$ than control group inmates $(M=0.16, S D=0.52), t(178)=15.60, p<.001, d=-2.06($ large effect size $)$.

A hierarchical multiple regression was used to assess the ability of psychological variables (mental health history, Psychoticism from the MMPI-2-RF) to predict Violence 
Severity Index (VSI) scores, after controlling for demographic variables (age, race) and criminal variables (conviction offense, sentence). Preliminary analyses were conducted to ensure there was no violation of the assumptions of normality, linearity, multicollinearity, and homoscedasticity. All assumptions were satisfied. Table 9 presents the results of the multiple regression analysis. Age and race (white, non-white) were entered at Step 1, explaining 11.2\% of the variance in VSI scores. An additional $6.6 \%$ of variance was accounted for after entering conviction offense and sentence length. A total of $17.8 \%$ of variance was accounted for with the variables entered at both Step 1 and 2. After entering mental health history and Psychoticism as measured on the MMPI-2-RF at Step 3, the total variance explained by the model as a whole was $20.2 \%, F(6,166)=8.27, p<.001$. Mental health history and the Psychoticism scale explained an additional $3.3 \%$ of the variance in VSI scores, after controlling for demographic and criminal variables, $R$ squared change $=0.03, F$ change $(2,166)=3.58, p=.030$. In the final model, all but two variables entered were statistically significant predictors of VSI scores. Age, race, sentence, and mental health history were significant predictors. Conviction offense and psychoticism were not significant in the full model.

Purpose 3-Cluster Analysis of Violent Inmates. Lastly, a cluster analysis was conducted for the violent inmate group $\left(n=55^{4}\right)$ using the five scales on the MMPI-2-RF that measure psychopathologies among personality constructs. These five scales include Aggressiveness, Psychoticism, Disconstraint, Negative Emotionality/Neuroticism, and Introversion/Low Positive Emotionality. A hierarchical cluster analysis using Ward's method (Ward, 1963) was used, as this method forms clusters with minimal variance within the clusters (Blashfield, 1976). In the process of running the cluster analysis, scores on the five MMPI-2-RF

\footnotetext{
${ }^{4}$ Five inmates in the violent group refused to complete the MMPI-2-RF and could not be included in the cluster analysis.
} 
scales were converted to z-scores to standardize comparisons. Three clusters were produced using Ward's method of hierarchical cluster analysis. Analysis of the agglomeration schedule indicated that after three clusters, subsequent clusters made very little additional distinction between cases. Two clusters seemed to group variables that were still fairly distinct from one another, making the optimal number of clusters three. A subsequent cluster analysis was run with three clusters, which placed each violent inmate into one of three clusters based on their scores on the five MMPI-2-RF scales. A new variable was created based on this cluster analysis that identified which of the three clusters the inmate was placed in (Cluster 1, 2, or 3). This new variable was used in subsequent univariate analyses.

A one-way analysis of variance was run to determine if the clusters significantly differed on any of the five MMPI-2-RF personality scales. These results are presented in Table 10. Results showed that four of the five personality scales were significantly different based upon the three clusters and using Bonferroni corrections $(p=.01)$. Cluster 1 of violent inmates endorsed significantly more symptoms of Psychoticism and Negative Emotionality/Neuroticism than inmates in the other two clusters. Cluster 2 inmates were primarily high on the scales of Aggressiveness and Disconstraint, whereas Cluster 3 inmates were violent, but scored significantly lower on the four personality scales (Aggressiveness, Psychoticism, Disconstraint, Negative Emotionality/Neuroticism). Although the finding was not statistically significant with Bonferroni corrections, Cluster 3 inmates scored moderately on the scale of Low Positive Emotionality/Introversion. Cluster 1 inmates were best characterized by their endorsement of psychotic symptoms and worry. Cluster 2 inmates were predominately aggressive and impulsive. Cluster 3 inmates were lower on scales assessing active psychotic and neurotic symptomology, 
as well as aggressive acting-out behaviors. Figure 1 displays the results of each MMPI-2-RF personality scale for the three clusters.

Although this purpose was exploratory, Cluster 1 supports the speculated hypothesis that some violent inmates would have higher scores on Psychoticism. The higher scores on Negative Emotionality/Neuroticism for this cluster was not hypothesized. Cluster 2 also coincides with the hypothesis that some violent inmates would be higher in Aggression and Disconstraint. There was no a priori hypothesis regarding a third cluster of inmates and how these inmates would differ on the MMPI-2-RF.

\section{Prototypes of Clusters}

Demographic, criminal, and psychological variables for the three clusters were examined and revealed some interesting patterns. These characteristics are described in the following prototypical descriptions of the three clusters. Table 11 shows differences for categorical demographic, criminal, and psychological variables among the three clusters. Table 12 presents the differences among the three clusters for continuous variables.

The inmates in Cluster 1 (primarily defined by high scores on Psychoticism and Negative Emotionality/Neuroticism) were incarcerated at a significantly older age ( $M=31.00$, $S D=11.31)$ than inmates in Cluster $2(M=23.20, S D=4.41), F(2,52)=3.03, p=.05$, partial $\eta^{2}=.10$ (medium effect size). Inmates in Cluster 1 were significantly more likely to require special education services in school than inmates in the other two clusters, $\chi^{2}(2, N=60)=6.36, p$ $=.042, \varphi=0.35$ (medium effect size). They also had significantly lower IQ scores $(M=81.00$, $S D=21.41)$ than the other two clusters of violent inmates, $F(2,51)=3.62, p=.03$, partial $\eta^{2}=$ .12 (large effect size). Most of these inmates had a history of mental health problems (81.8\%), although this difference was not statistically significant. Inmates in this cluster were significantly 
more likely to be convicted of a sexual offense, $\chi^{2}(2, N=60)=15.34, p=0.05, \varphi_{c}=0.37$ (medium effect size) than inmates in the other two clusters. In addition to their MMPI-2-RF scores, inmates in this cluster also can be characterized by their older age, low IQ, history of special education services, and conviction of a sexual offense.

The inmates in Cluster 2 (primarily defined by high scores on Aggressiveness and Disconstraint) were incarcerated at the youngest age $(M=23.20, S D=4.41)$ of the three groups, $F(2,52)=3.03, p=.05$, partial $\eta^{2}=.10$ (medium effect size). This group had the greatest variety of types of crime leading to their current incarceration with the most frequent being violent crime $(n=7,46.7 \%)$ and the next most common conviction offenses being property ( $n=$ $3,20.0 \%)$ and drug $(n=3,20.0 \%)$ crimes. They were significantly more likely to be incarcerated for a drug or "other" crime than inmates in Cluster 1 or $3, \chi^{2}(2, N=60)=15.34, p=0.05, \varphi_{c}=$ 0.37 (medium effect size). This cluster has younger inmates with greater variety of conviction offenses.

The inmates in Cluster 3 (primarily defined by low scores on four of the five personality scales on the MMPI-2-RF, and moderate scores on Introversion/Low Positive Emotionality) had an average age of incarceration of 27.79 years $(S D=8.36)$, which is older than inmates in Cluster 2 and younger than inmates in Cluster 1. Although a trend-level finding, these inmates were sentenced to longer durations $(M=11.69,11.78)$ than inmates in the other two clusters, $F(2,52)=2.84, p=.07$, partial $\eta^{2}=.10$ (medium effect size). They were predominately convicted of violent crimes (55.2\%) and sexual offenses (24.1\%). Cluster 3 inmates also scored significantly higher than the other two clusters on the Beta-III with an average IQ score of 96.90 $(S D=17.05), F(2,51)=3.62, p=.03$, partial $\eta^{2}=.12$ (medium effect size). Cluster 3 inmates also were the least likely to have a history of special education services. 
Clusters did not differ on the continuous measure of Violence Severity Index (VSI) scores. When the frequencies of VSI scores were examined, results indicated scores for Cluster 1 inmates ranged from one to four, Cluster 2 inmates ranged from one to eight, and Cluster 3 inmates ranged from one through ten on the VSI. There were no significant differences when frequencies of VSI scores were examined for the three clusters.

\section{Discussion}

This study yielded several noteworthy findings. Univariate analyses indicated that violent inmates were more frequently non-white, incarcerated at a younger age, convicted of a violent crime, sentenced to a longer period of incarceration, and more frequently had a history of mental illness than control group inmates. Although violent and control group inmates had equivalent military histories, violent inmates were significantly more likely to be dishonorably discharged than control group inmates who served in the military. Another mark of defiant behaviors was found when examining inmates' approach to psychological testing. Significantly more violent inmates refused to complete the MMPI-2-RF, and of those who did complete the measure, violent inmates had significantly more invalid protocols than control group inmates. Specifically, every inmate who refused to complete the MMPI-2-RF was in the violent group. Perhaps this blatant refusal to complete this measure could be an easy, useful way to accurately identify those problematic, violent inmates from the general population of inmates. Approach to testing is an interesting marker of defiance and requires additional research to fully understand the utility of this behavior in predicting violent inmates. VSI scores were calculated for all inmates who engaged in physical violence towards other inmates or staff while incarcerated, and not surprisingly, violent inmates had significantly higher scores on this weighted measurement of violence than the control group inmates. 
A logistic regression analysis found that age, mental health history, and the MMPI-2-RF scale of Psychoticism were significant predictors in identifying violent from control inmates. The entire model with demographic (age, race), criminal (crime, sentence length), and psychological variables (mental health history, Psychoticism) entered was able to accurately predict group membership for $73.4 \%$ of cases. The psychological variables of mental health history and the Psychoticism scale improved the predictive utility of the model by an additional $1.1 \%$, which although small, was statistically significant and supported previous findings that inmates with a mental health history were more likely to be violent while incarcerated (Toch \& Kupers, 2007; Walters, 2011; Walters \& Crawford, 2013). Furthermore, the logistic regression accurately predicted $40.0 \%$ of violent inmates being in the violent group with all variables entered. Prediction rates for violent inmates were $14.5 \%$ with only demographic variables and $30.9 \%$ accuracy with demographic and criminal variables. Psychological variables increased the accuracy of accurately predicting violent inmates from control inmates. Using the MMPI-2-RF scale of Psychoticism to predict violent from control group inmates is a new contribution to this area of research.

Using the weighted VSI score as the continuous criterion variable in a hierarchical multiple regression, age, race, sentence length, and mental health history were all found to be significant predictors of VSI scores. With all variables entered, the model accounted for $20 \%$ of the variance in VSI scores. Mental health history was a strong predictor of VSI scores in the final model; however, Psychoticism was unable to significantly predict VSI scores.

Using the MMPI-2-RF Personality Psychopathology scales for a hierarchical cluster analysis, three distinct groups of inmates were identified among the violent inmates in this sample. One group was higher in traits of Psychoticism and Negative Emotionality/Neuroticism. 
This cluster of inmates endorsed more symptoms of paranoia and psychotic beliefs/experiences, as well as excessive levels of worry and self-doubt. Another group was primarily characterized by their aggressive, impulsive, and risk-taking tendencies. The third and largest group of inmates endorsed the fewest items of Aggressiveness, Disconstraint, Negative Emotionality/Neuroticism, and Psychoticism. It seems these inmates had less aggressive propensities with fewer mental health needs, but perhaps acted violently in an effort to protect themselves or create a reputation as someone not to be crossed. These clusters suggest there may be a distinction between the inmates who act violently out of aggression and impulsivity, compared to inmates who have more significant mental health needs and those inmates who are just trying to keep to themselves and survive prison.

\section{Non-Statistically Significant Findings}

Although many noteworthy findings resulted from this study, there also was a lack of some results which was unexpected or incongruent with previous research. For example, in the current study there was no significant difference between the two inmate groups on both variables of education and IQ. Previous research has consistently found that the more education the inmate has attained, the less likely they are to engage in violence while incarcerated (Berg \& DeLisi, 2006; Cunningham, Sorensen, \& Reidy, 2005; DeLisi, Berg, \& Hochstetler, 2004). This finding was not replicated in the current study; however, the imperfections between years of education and actual intelligence were considered. Diamond, Morris, and Barnes (2012) found as an inmate's IQ increased (as measured on the WAIS-R), their likelihood of being involved in violence decreased. The current study was unable to replicate those results using the Beta-III, a brief measure of nonverbal intelligence; IQ scores on this measure did not significantly differ between the violent and control group inmates. A study using a more thorough, up-to-date 
intelligence assessment, such as the Wechsler Assessment of Intelligence Scale-IV, would be helpful to clarify the relation between IQ and involvement in prison violence.

Although substance abuse has been researched previously, its relationship to prison violence has either not been compared to a group of non-violent inmates (Friedmann, Melnick, Jiang, \& Hamilton, 2008) or was studied based on self-reported perpetration of violence and in conjunction with mental illness, and not independently (Wood, 2012; Wood \& Buttaro, 2013). The inmates in the current study did not significantly differ in their self-reported substance abuse history, as measured on the TCU Drug Screen II. This was the first study to independently investigate the relationship between having a history of substance abuse and prison violence. Additional research would help clarify if a relationship truly exists between these two variables or not.

The current study also did not find significant differences between the violent and control group inmates on the Cognitive Complaints scale on the MMPI-2-RF. This scale was investigated based on a previous study that found inmates who were rated higher on the variable of confusion/memory impairment, were more likely to be violent while incarcerated (Baskin, Sommers, \& Steadman, 1991). The lack of replication of this finding in the current study could be due to the variable being assessed very differently in the two studies. Specifically, the MMPI2-RF Cognitive Complaints scale was used in this study, compared to case manager ratings of observed inmates' behaviors on a one to five Likert scale in Baskin, Sommers, and Steadman's study (1991). Further research could better clarify this relationship, as there is anecdotal evidence to suggest that inmates who experience memory impairments and confusion can be more hostile, unpredictable, and potentially aggressive. Also considering the aging inmate 
population (Motivans, 2013), the relationship between memory impairment and prison violence will need more attention.

Previous research found predictive utility in self-report measures of aggressiveness for identifying violent from non-violent inmates. Even with five different scales on the MMPI-2-RF tapping into the construct of aggression, no significant differences were found between the two inmate groups in this study. Of the three studies that found predictive utility in the variable of aggression (Gillespie, 2005; Lahm, 2008; Lahm, 2009), researchers used some variation of Buss and Perry's Aggression Questionnaire with different reasons for each alteration of this measure. Using the Personality Assessment Inventory, Walters, Duncan, and Geyer (2003) initially reported significant results on the Aggression scale, but this finding did not persist once Bonferroni corrections were applied. Given the methodological concerns of previous research reporting significant results and the lack of results of more methodologically-sound studies, additional research is needed. Conceptually, it seems logical that more aggressive inmates would be more violent while incarcerated. Perhaps using the entire Buss and Perry’s Aggression Questionnaire or another measure of only aggression (compared to single, specific scales on a personality assessment measure) would better clarify this relation.

\section{Limitations}

Although this study found important results with implications to improve the prediction of inmates who will engage in prison violence, there were limitations to note. First, there is limited generalizability of the study results. The files from only one medium-security prison in the state of West Virginia were reviewed. Because of this limited sample, we are unable to determine if the same results would be found in a prison with a different security level, different inmates, or in a different state male inmates sentenced to any duration of time except Life 
Without Parole (sentences of Life with the possibility of Parole were included in this sample). Not being able to study this unique group of inmates sentenced to die in prison limits the generalizability of results, as previous research has been able to study this unique group and their propensity for prison violence in comparison to other inmates. To compensate for this limitation, sentence length was studied as a continuous variable based on the minimum time incarcerated before the inmate would be eligible for parole. This is a unique contribution to the literature as the majority of previous research examined sentence length categorically. Also, the geographic location of the prison in this study further limited the results. The demographic composition of HCC is likely very different from a prison in California. As a result, certain variables were simplified (e.g., race to categories of white and non-white) and other variables could not be examined at all (e.g., gang membership) because of the demographic composition of the state and its prisons.

Another limitation of this study was sample size. Only inmates designated as an "Assaultive" risk based on one serious act or repeated acts of prison violence comprised the violent group. Because of this conservative decision, there were only 60 inmates that met this criterion over the three month period of data collection. However, this group engaged in serious acts of violence that jeopardized the safety of the institution and injured the victim of their aggression. Thirty of the violent inmates only assaulted other inmates and 16 violent inmates only assaulted staff, whereas ten inmates from this group had both staff and inmate victims. To ensure a more representative sample of the remaining general population inmates, twice as many inmates were randomly selected for the control group. However, the variables that could be entered into the logistic and multiple regressions were still limited by sample size. More predicting variables could have been examined with a larger sample. 
As described in the introduction of this study, there are strengths and weaknesses to relying on official documentation of violent behavior instead of self-report data. These costs and benefits were recognized and carefully considered. It was ultimately decided that official documentation would be the more conservative, and ideally, accurate source of information; however, this method is not without faults. Underreporting is a primary drawback to relying solely on official records. It is likely that inmates engaged in other acts of violence that went unnoticed or unreported to staff. There also is a possibility of selectivity bias, in which staff favor particular inmates and overlook their misbehaviors, or staff unnecessarily target inmates they dislike. Relatedly, official documentation relies on how the staff write-up the incident report. It is possible that a staff member could over-exaggerate behaviors to "trump up" the charge, or minimize the inmate's actions from what actually happened. Staff also write-up an incident based on the information they have available to them. It is possible that an inmate acting purely in self-defense could be perceived as the aggressor and sanctioned for engaging in prison violence according to the staff member's perspective of the incident. Equally possible is the scenario of a busy staff person who chooses not to write-up the violation all together to avoid the time-consuming paperwork and to not get involved in the disciplinary process.

\section{Study Implications and Future Directions}

To date, this study was the first to utilize the MMPI-2-RF in an effort to predict those inmates who will engage in prison violence. Particularly interesting and novel was the use of the five Personality Psychopathology scales on the MMPI-2-RF to identify distinct clusters of violent inmates. Additional research would benefit from testing the validity of these clusters with a different sample of inmates to enhance generalizability. Because the MMPI-2-RF is a newer version of the widely accepted and utilized MMPI-2, more research will continue to be necessary 
to fully understand the various implications for this measure among incarcerated individuals. As a result of the sample size of the current study, only theoretically derived MMPI-2-RF scales could be analyzed. It is possible that other scales on the MMPI-2-RF could significantly predict prison violence. This possibility should be further evaluated with future research using a larger, more diverse sample.

Military history was an exploratory variable in this study. Involvement in the military did not differ between violent and control group inmates, but how they were discharged from the military did differ. Violent inmates were much more likely to be dishonorably discharged from the military than control group inmates. Although both groups could not behave appropriately to live in society after their military involvement, those dishonorably discharged seem to have a pattern of inappropriate behaviors in the military, in society, and in prison. Dishonorable military discharge may be a quick and easy screener for prison staff to identify inmates that are at an increased risk for prison violence. Given the small number of inmates that had a history of being in the military in this study, additional research will be necessary to better understand the utility of this variable for identifying inmates prone to violence.

Additional research to further investigate the motives for inmates to engage in prison violence would be advantageous. It would be interesting and informative to know from the inmates' perspective what prompted their violence. Combining the perspective of the inmate with the three distinct clusters of violent inmates that resulted in this study, would create a more complete picture of the motivating events prompting violent behaviors. It also would take into consideration the perspective of staff, who identified the inmate as violent and documented the violent act, and the inmates' account of the situation and their motive. 
Considering the overwhelming number of adults incarcerated in the United States, prisons continue to be crowded environments ripe for violence. This study demonstrated that violent inmates have some shared characteristics that significantly differ from inmates that are not violent while incarcerated. Although a challenging task, it is necessary to better understand and be able to more accurately predict the individuals who will engage in prison violence to enhance the safety of staff working within the institution and other inmates living in that environment. 


\section{References}

American Psychiatric Association. (2013). Diagnostic and statistical manual of mental disorders (5th ed.). Arlington, VA: American Psychiatric Publishing.

Baskin, D. R., Sommers, I., \& Steadman, H. J. (1991). Assessing the impact of psychiatric impairment on prison violence. Journal of Criminal Justice, 19, 271-280.

Ben-Porath, Y. S. (2012). Introducing the MMPI-2-RF. Presented as a webinar with slides retrieved from http://www.pearsonassessments.com/hai/images/pdf/webinar/2012-mmpi2-rf.pdf

Ben-Porath, Y. S. \& Tellegen, A. (2008). MMPI-2-RF (Minnesota multiphasic personality inventory-2): Manual for administration, scoring, and interpretation. Minneapolis, $\mathrm{MN}$ : University of Minnesota Press.

Berg, M. T. \& DeLisi, M. (2006). The correctional melting pot: Race, ethnicity, citizenship, and prison violence. Journal of Criminal Justice, 34, 631-642. doi: 10.1016/j.jcrimjus. 2006.09.016

Blashfield, R. K. (1976). Mixture model tests of cluster analysis: Accuracy of four agglomerative hierarchical methods. Psychological Bulletin, 83(3), 377-388. doi: 10.1037/00332909.83.3.377

Blitz, C. L., Wolff, N., \& Shi, J. (2008). Physical victimization in prison: The role of mental illness. International Journal of Law and Psychiatry, 31, 385-393. doi: 10.1016/j.ijlp.2008.08.005

Bryne, J. M. \& Hummer, D. (2007). Myths and realities of prison violence: A review of the evidence. Victims \& Offenders, 2(1), 77-90. doi: 10.1080/15564880601087241 
Carson, E. A. \& Golinelli, D. (2013). Prisoners in 2012-Advance counts (Bureau of Justice Statistics Bulletin NCJ 242467). Retrieved from the U.S. Department of Justice, Office of Justice Programs, Bureau of Justice Statistics website: http://www.bjs.gov/content/pub /pdf/p12ac.pdf

Clegg, C., Fremouw, W., Horacek, T., Cole, A., \& Schwartz, R. (2010). Factors associated with treatment acceptance and compliance among incarcerated male sex offenders. International Journal of Offender Therapy and Comparative Criminology, 55(6), 880897. doi: 10.1177/0306624X10376160

Cunningham, M. D. \& Sorensen, J. R. (2006a). Nothing to lose? A comparative examination of prison misconduct rates among life-without-parole and other long-term high-security inmates. Criminal Justice and Behavior, 33(6), 683-705. doi:

$10.1177 / 0093854806288273$

Cunningham, M. D. \& Sorensen, J. R. (2006b). Actuarial models for assessing prison violence risk: Revisions and extensions of the risk assessment scale for prison (RASP). Assessment, 13(3), 253-265. doi: 10.1177/1073191106287791

Cunningham, M. D. \& Sorensen, J. R. (2007). Predictive factors for violent misconduct in close custody. The Prison Journal, 87(2), 241-253. doi: 10.1177/0032885507303752

Cunningham, M. D., Sorensen, J. R., \& Reidy, T. J. (2005). An actuarial model for assessment of prison violence risk among maximum security inmates. Assessment, 12(1), 40-49. doi: $10.1177 / 1073191104272815$

Cunningham, M. D., Sorensen, J. R., Vigen, M. P., \& Woods, S. O. (2010). Inmate homicides: Killers, victims, motives, and circumstances. Journal of Criminal Justice, 38, 348-358. doi: 10.1016/j.jcrimjus.2010.03.008 
DeLisi, M. (2003). Criminal careers behind bars. Behavioral Sciences and the Law, 21, 653-669. doi: 10.1002/bsl.531

DeLisi, M., Berg, M. T., \& Hochstetler, A. (2004). Gang members, career criminals and prison violence: Further specification of the importation model of inmate behavior. Criminal Justice Studies, 17(4), 369-383. doi:10.1080/1478601042000314883

Diamond, B., Morris, R. G., \& Barnes, J. C. (2012). Individual and group IQ predict inmate violence. Intelligence, 40, 115-122. doi: 10.1016/j.intell.2012.01.010

Duhart, D. T. (2001). Violence in the workplace, 1993-99 (Bureau of Justice Statistics Special Report NCJ 190076). Retrieved from the U.S. Department of Justice, Office of Justice Programs, Bureau of Justice Statistics website: http://bjs.ojp.usdoj.gov/content/pub/pdf/ vw99.pdf

Edens, J. F., Poythress, N. G., Lilienfeld, S. O., Patrick, C. J., \& Test, A. (2008). Further evidence of the divergent correlates of the psychopathic personality inventory factors: Prediction of institutional misconduct among male prisoners. Psychological Assessment, 20(1), 86-91.

Friedmann, P. D., Melnick, G., Jiang, L., \& Hamilton, Z. (2008). Violent and disruptive behavior among drug-involved prisoners: Relationship with psychiatric symptoms. Behavioral Sciences and the Law, 26, 389-401. doi: 10.1002/bsl.824

Gadon, L., Johnstone, L., \& Cooke, D. (2006). Situational variables and institutional violence: A systematic review of the literature. Clinical Psychology Review, 26, 515-534. doi: 10.1016/j.cpr.2006.02.002

Gillespie, W. (2005). Racial differences in violence and self-esteem among prison inmates. American Journal of Criminal Justice, 29(2), 161-185. doi: 10.1007/BF02885734 
Glaze, L. E. \& Herberman, E. J. (2013). Correctional populations in the United States, 2012 (Bureau of Justice Statistics Bulletin NCJ 243936). Retrieved from the U.S. Department of Justice, Office of Programs, Bureau of Justice Statistics website: http://www.bjs.gov /content/pub/pdf/cpus12.pdf

Graham, J. R. (2011). MMPI-2: Assessing personality and psychopathology $\left(3^{\text {rd }}\right.$ ed.). New York, NY: Oxford.

Griffin, M. L. \& Hepburn, J. R. (2006). The effect of gang affiliation on violent misconduct among inmates during the early years of confinement. Criminal Justice and Behavior, 33(4), 419-448. doi: 10.1177/0093854806288038

Harer, M. D. \& Steffensmeier, D. J. (1996). Race and prison violence. Criminology, 34(3), 323355. doi: 10.1111/j.1745-9125.1996.tb01210.x

Hildebrand, R. (2013). WV division of corrections annual report 2013. Retrieved from the West Virginia Division of Corrections website: http://www.wvdoc.com/wvdoc/Officeof ResearchPlanning/tabid/70/Default.aspx

International Centre for Prison Studies (2010, March). Prison brief: Highest to lowest rates.

Retrieved from King's College London School of Law website: http://www.webcitation.org/5xRCN8YmR

James, D. J. \& Glaze, L. E. (2006). Mental health problems of prison and jail inmates (Bureau of Justice Statistics Special Report NCJ 213600). Retrieved from the U.S. Department of Justice, Office of Justice Programs, Bureau of Justice Statistics website: http://www.bjs.gov/content/pub/pdf/mhppji.pdf

Kellogg, C. E. \& Morton, N. W. (1999). Beta-third edition (Beta-III). Sydney, NWS: Person Corporation. 
Knight, K., Simpson, D. D., \& Morey, J. T. (2002). TCU-NIC cooperative agreement: Final report. Fort Worth: Texas Christian University, Institute of Behavioral Research. U.S. Department of Justice Grant: 99-MU-MU-K008. Retrieved from: https://www.ncjrs.gov/pdffiles1/nij/grants/196682.pdf

Lahm, K. F. (2008). Inmate-on-inmate assault: A multilevel examination of prison violence. Criminal Justice and Behavior, 35, 120-137. doi: 10.1177/0093854807308730

Lahm, K. F. (2009). Inmate assaults on prison staff: A multilevel examination of an overlooked form of prison violence. The Prison Journal, 89, 131-150. doi: 10.1177/00328855 09334743

Macmanus, D. \& Wessely, S. (2012). Trauma, psychopathology, and violence in recent combat veterans. In C. S. Widom (Ed.), Trauma, psychopathology, and violence: Causes, consequences, or correlates? (pp. 267-287). New York, NY: Oxford.

Marion, B. E., Sellbom, M., Salekin, R. T., Toomey, J. A., Kucharski, L. T., \& Duncan, S. (2013). An examination of the association between psychopathy and dissimulation using the MMPI-2-RF validity scales. Law and Human Behavior, 37(4), 219-230. doi: 10.1037/lhb0000008

Megargee, E. I. (1994). Using the Megargee MMPI-based classification system with MMPI-2s of male prison inmates. Psychological Assessment, 6(4), 337-344. doi: 10.1037/10403590.6.4.337

Megargee, E. I. (1997). Using the Megargee MMPI-based classification system with the MMPI2s of female prison inmates. Psychological Assessment, 9(2), 75-82. doi: 10.1037/10403590.9 .2 .75 
Megargee, E. I., Mercer, S. J., \& Carbonell, J. L. (1999). MMPI-2 with male and female state and federal prison inmates. Psychological Assessment, 11(2), 177-185.

Morey, L. C. (2007). The personality assessment inventory professional manual. Lutz, FL: Psychological Assessment Resources.

Motivans, M. (2013). Federal justice statistics, 2010 (Bureau of Justice Statistics Special Report NCJ 239913). Retrieved from the U.S. Department of Justice, Office of Justice Programs, Bureau of Justice Statistics website: http://www.bjs.gov/content/pub/pdf/fjs10.pdf

Mumola, C. J. (2000). Veterans in prison or jail (Bureau of Justice Statistics Special Report NCJ 178888). Retrieved from the U.S. Department of Justice, Office of Justice Programs, Bureau of Justice Statistics website: http://amador.networkofcare.org/library/vpj\%20 Veterans\%20in\%20Prison\%20or\%20Jail.pdf

Mumola, C. J. (2005). Suicide and homicide in state prisons and local jails (Bureau of Justice Statistics Special Report NCJ 210036). Retrieved from the U.S. Department of Justice, Office of Justice Programs, Bureau of Justice Statistics website: http://bjs.ojp.usdoj.gov/content/pub/pdf/shsplj.pdf

Nandy, K. (2012). Understanding and quantifying effect sizes [pdf document]. Retrieved from http://nursing.ucla.edu/workfiles/research/Effect\%20Size\%204-9-2012.pdf

The National Center on Addiction and Substance Abuse at Columbia University (2010, February). Behind bars II: Substance abuse and American's prison population. Retrieved from the National Center on Addiction and Substance Abuse (CASA) website: http:// www.casacolumbia.org/addiction-research/reports/substance-abuse-prison-system-2010 Neisser, U. (1997). Rising scores on intelligence test. American Scientist, 85, 440-477. 
Noonan, M. E. (2013). Mortality in local jails and state prisons, 2000-2011-Statistics tables (Bureau of Justice Statistics Special Report NCJ 242186). Retrieved from the U.S. Department of Justice, Office of Justice Programs, Bureau of Justice Statistics website: http://www.bjs.gov/content/pub/pdf/mljsp0011.pdf

Noonan, M. E. \& Mumola, C. J. (2007). Veterans in state and federal prison (Bureau of Justice Statistics Special Report NCJ 217199). Retrieved from the U.S. Department of Justice, Office of Justice Programs, Bureau of Justice Statistics website: http://www.bjs.gov/ content/pub/pdf/vsfp04.pdf\#page=1\&zoom=auto,0,792

Pearson Education, Incorporated (2012). Assessment and information: Beta-III. Retrieved from: http://www.pearsonassessments.com/HAIWEB/Cultures/enus/Productdetail.htm?Pid=01 5-8685-202\&Mode=summary

Peters, R. H., Greenbaum, P. E., Steinberg, M. L., Carter, C. R., Ortiz, M. M., Fry, B. C., \& Valle, S. K. (2000). Effectiveness of screening instruments in detecting substance use disorders among prisoners. Journal of Substance Abuse Treatment, 18(4), 349-358. doi: 10.1016/S0740-5472(99)00081-1

Quinsey, V. L., Harris, G. T., Rice, M. E., \& Cormier, C. A. (2006). Violent sex offenses: How are they best measured from official records? Law and Human Behavior, 30(4), 525-541. doi: 10.1007/s10979-006-9022-3

Schenk, A. M. \& Fremouw, W. J. (2012). Individual characteristics related to prison violence: A critical review of the literature. Aggression and Violent Behavior, 17(5), 430-442. doi: 10.1016/j.avb.2012.05.005 
Sellbom, M. \& Bagby, R. M. (2008). Validity of the MMPI-2-RF (restructured form) L-r and K-r scales in detecting underreporting in clinical and nonclinical samples. Psychological Assessment, 20(4), 370-376. doi: 10.1037/a0012952

Sellbom, M., Ben-Porath, Y. S., Patrick, C. J., Wygant, D. B., Gartland, D. M. \& Stafford, K. P. (2012). Development and construct validation of MMPI-2-RF indices of global psychopathy, fearless-dominance, and impulsive-antisociality. Personality Disorders: Theory, Research, and Treatment, 3(1), 17-38. doi: 10.1037/a0023888

Shaw, R. G. \& Mitchell-Olds, T. (1993, September). Anova for unbalanced data: An overview. Ecology, 74(6), 1638-1645.

Stephan, J. J. (2008). Census of state and federal correctional facilities, 2005 (Bureau of Justice Statistics Special Report NCJ 222182). Retrieved from the U.S. Department of Justice, Office of Justice Programs, Bureau of Justice Statistics website: http://bjs.ojp.usdoj.gov/content/pub/pdf/csfcf05.pdf

Stephan, J. J. \& Karberg, J. C. (2003). Census of state and federal correctional facilities, 2000 (Bureau of Justice Statistics Special Report NCJ198272). Retrieved from the U.S. Department of Justice, Office of Justice Programs, Bureau of Justice Statistics website: http://www.bjs.gov/content/pub/pdf/csfcf00.pdf

Sorensen, J. \& Cunningham, M. D. (2008). Conviction offense and prison violence: A comparative study of murders and other offenders. Crime \& Delinquency, 56(1), 103 125. doi: $10.1177 / 0011128707307175$

Sorensen, J. R., Cunningham, M. D., Vigen, M. P., \& Woods, S. O. (2011). Serious assaults on prison staff: A descriptive analysis. Journal of Criminal Justice, 39, 143-150. doi: 10.1016/j.jcrimjus2011.01.002 
Tabachnick, B. G. \& Fidell, L. S. (2007). Using multivariate statistics, $5^{\text {th }}$ edition. Boston, MA: Pearson Education, Inc.

Tarescavage, A. M., Wygant, D. B., Boutacoff, L. I., \& Ben-Porath, Y. S. (2013). Reliability, validity, and utility of the Minnesota Multiphasic Personality Inventory-2-Restructured Form (MMPI-2-RF) in assessments of bariatric surgery candidates. Psychological Assessments, 25(4), 1179-1195. doi:10.1037/a0033694

Tellegen, A. \& Ben-Porath, Y. S. (2008). MMPI-2-RF: Technical manual. Minneapolis: University of Minnesota Press.

Thomas, M. L. \& Locke, D. E. C. (2010). Psychometric properties of the MMPI-2-RF somatic complaints (RC1) scale. Psychological Assessment, 22(3), 492-503. doi:

$10.1037 / \mathrm{a} 0019229$

Toch, H. \& Kupers, T. A. (2007). Violence in prisons, revisited. Journal of Offender Rehabilitation, 45(3-4), 1-28. doi: 10.1300/J076v45n03_01

Unified Crime Report (n.d.). Crime in the United States by volume and rate per 100,000 inhabitants, 1993-2012. Retrieved from the Federal Bureau of Investigations website: www.fbi.gov/about-us/cjis/ucr/crime-in-the-u.s/2012/crime-in-the-u.s.-2012/tables/ 1tabledatadecoverviewpdf/table_1_crime_in_the_united_states_by_volume_and_rate_per _100000_inhabitants_1993-2012.xls

Unified Crime Report (2010). Crime in the United States. Retrieved from the U.S. Department of Justice-Federal Bureau of Investigation website at http://www.fbi.gov/aboutus/cjis/ucr/crime-in-the-u.s/2010/crime-in-the-u.s.-2010/persons-arrested 
Van Der Heijden, P. T., Egger, J. I. M., Derksen, J. J. L. (2010). Comparability of scores on the MMPI-2-RF scales generated with the MMPI-2 and MMPI-2-RF booklets. Journal of Personality Assessment, 92(3), 254-259. doi: 10.1080/00223891003670208

Walters, G. D. (2011). Criminal thinking as a mediator of the mental illness-prison violence relationship: A path analytic study and causal mediation analysis. Psychological Services, 8(3), 189-199. doi: 10.1037/a0024684

Walters, G. D. \& Crawford, G. (2013, October 14). Major mental illness and violence history as predictors of institutional misconduct and recidivism: Main and interaction effects. Law and Human Behavior. Advanced online publication. doi: 10.1037/lhb0000058

Walters, G. D., Duncan, S. A., \& Geyer, M. D. (2003). Predicting disciplinary adjustment in inmates undergoing forensic evaluation: A direct comparison of the PCL-R and the PAI. The Journal of Forensic Psychiatry \& Psychology, 14(2), 382-393. doi: $10.1080 / 1478994031000136527$

Ward, J. (1963). Hierarchical grouping to optimize an objective function. Journal of the American Statistical Association, 58, 236-244.

Wolff, N., Blitz, C. L., Shi, J., Siegel, J., \& Bachman, R. (2007). Physical violence inside prisons: Rates of victimization. Criminal Justice and Behavior, 34(5), 588-599. doi: $10.1177 / 0093854806296830$

Wolff, N., Shi, J., \& Bachman, R. (2008). Measuring victimization inside prisons: Questioning the questions. Journal of Interpersonal Violence, 23(10), 1343-1362. doi: $10.1177 / 0886260508314301$

Wood, S. R. (2012). Dual severe mental and substance use disorders as predictors of federal inmate assaults. The Prison Journal, 93(1), 34-56. doi: 10.1177/0032885512467312 
Wood, S. R. \& Buttaro, A. (2013). Co-occurring severe mental illnesses and substance abuse disorders as predictors of state prison inmate assaults. Crime \& Delinquency, 59(4), 510535. doi: 10.1177/0011128712470318

Wygant, D. B., Sellbom, M., Gervais, R. O., Ben-Porath, Y. S., Stafford, K. P., Freeman, D. B., \& Heilbronner, R. L. (2010). Further validation of the MMPI-2 and MMPI-2-RF response bias scale: Findings from disability and criminal forensic settings.

Psychological Assessment, 22(4), 745-756. doi: 10.1037/a0020042 
Table 1

Initial Kappa Statistics for Interrater Reliability of Dichotomous and Categorical Variables

Variable

Race

History of Special Education

History of Military Service

Current Offense Category

History of Prior Criminal Convictions

Prior Prison Sentence

Mental Health History

Prescribed Psychotropic Medications

History of Suicide Attempts

MMPI-2-RF Results Valid

MMPI-2-RF Refused
Initial Kappa Value
0.87

1.00

1.00

0.93

0.82

0.86

1.00

0.92

1.00

1.00

Note. All discrepancies in rating were resolved by the primary rater and interrater reviewing the file, identifying, and agreeing on the accurate data together. 
Table 2

Initial Interrater Reliability of Continuous Variables

\begin{tabular}{|c|c|}
\hline Variable & $\begin{array}{c}\text { Intraclass Correlation Coefficient } \\
\text { (ICC) }\end{array}$ \\
\hline Age at Incarceration & 1.00 \\
\hline Age at Violent Incident & 1.00 \\
\hline Years of Education & 0.90 \\
\hline Number of Incident Reports & 1.00 \\
\hline Number of Violent Incident Reports & 0.92 \\
\hline Number of Potentially Violent Incident Reports & 1.00 \\
\hline \multicolumn{2}{|l|}{ MMPI-2-RF Scales } \\
\hline Cannot Say (CNS) & 1.00 \\
\hline Variable Response Inconsistency (VRIN) & 1.00 \\
\hline True-Response Inconsistency (TRIN) & 1.00 \\
\hline Infrequent Responses (Fr) & 1.00 \\
\hline Infrequent Psychopathology Responses (Fpr) & 1.00 \\
\hline Infrequent Somatic Responses (Fs) & 1.00 \\
\hline Symptom Validity (FBS) & 1.00 \\
\hline Response Bias (RBS) & 1.00 \\
\hline Uncommon virtues (Lr) & 1.00 \\
\hline Adjustment Validity (Kr) & 1.00 \\
\hline Emotional/Internalizing Dysfunction (EID) & 1.00 \\
\hline Thought Dysfunction (THD) & 1.00 \\
\hline
\end{tabular}


(Table 2 Continued)

1.00

Behavioral/Externalizing Dysfunction (BXD)

Demoralization (RCdem)

1.00

Somatic Complaints (RC1som)

1.00

Low Positive Emotions (RC2lpe)

1.00

Cynicism (RC3cyn)

Antisocial Behavior (RC4asb)

1.00

Ideas of Persecution (RC6per)

1.00

Dysfunctional Negative Emotions (RC7dne)

1.00

Aberrant Experiences (RC8abx)

1.00

Hypomanic Activation (RC9hpm)

1.00

Malaise (MLS)

1.00

Gastro-Intestinal Complaints (GIC)

Head Pain Complaints (HPC)

1.00

Neurological Complaints (NUC)

1.00

Cognitive Complaints (COG)

1.00

Suicidal/Death Ideation (SUI)

1.00

Helplessness/Hopelessness (HLP)

1.00

Self-Doubt (SFD)

1.00

Inefficacy (NFC)

1.00

Stress/Worry (STW)

1.00

Anxiety (AXY)

1.00

Anger Proneness (ANP)

1.00 
(Table 2 Continued)

1.00

Behavior-Restricting Fears (BRF)

$\begin{array}{lr}\text { Multiple Specific Fears (MSF) } & 1.00\end{array}$

$\begin{array}{lr}\text { Juvenile Conduct Problems (JCP) } & 1.00\end{array}$

$\begin{array}{ll}\text { Substance Abuse (SUB) } & 1.00\end{array}$

$\begin{array}{ll}\text { Aggression (AGG) } & .997\end{array}$

$\begin{array}{ll}\text { Activation (ACT) } & 1.00\end{array}$

$\begin{array}{ll}\text { Family Problems (FML) } & 1.00\end{array}$

$\begin{array}{lr}\text { Interpersonal Passivity (IPP) } & 1.00\end{array}$

$\begin{array}{lr}\text { Social Avoidance (SAV) } & 1.00\end{array}$

$\begin{array}{ll}\text { Shyness (SHY) } & 1.00\end{array}$

$\begin{array}{lr}\text { Disaffiliativeness (DSF) } & 1.00\end{array}$

$\begin{array}{ll}\text { Aesthetic-Literary Interests (AES) } & 0.99\end{array}$

$\begin{array}{lr}\text { Mechanical-Physical Interest (MEC) } & 1.00\end{array}$

$\begin{array}{ll}\text { Aggressiveness-Revised (AGGRr) } & 1.00\end{array}$

$\begin{array}{ll}\text { Psychoticism-Revised (PSYCr) } & 1.00\end{array}$

$\begin{array}{ll}\text { Disconstraint-Revised (DISCr) } & 0.74\end{array}$

Negative Emotionality/Neuroticism-Revised (NEGEr) 1.00

Introversion/Low Positive Emotionality-Revised (INTRr) $\quad 1.00$

$\begin{array}{ll}\text { Texas Christian University Total Score } & 0.95\end{array}$

Beta-III Intelligence Quotient Score $\quad 1.00$

Note. MMPI-2-RF = Minnesota Multiphasic Personality Inventory-2-Restructured Form. All discrepancies in scoring were resolved by the primary rater and interrater reviewing the file, identifying, and agreeing on the accurate data together. 
Table 3

Descriptive Statistics of Categorical Demographic, Criminal, and Psychological Variables

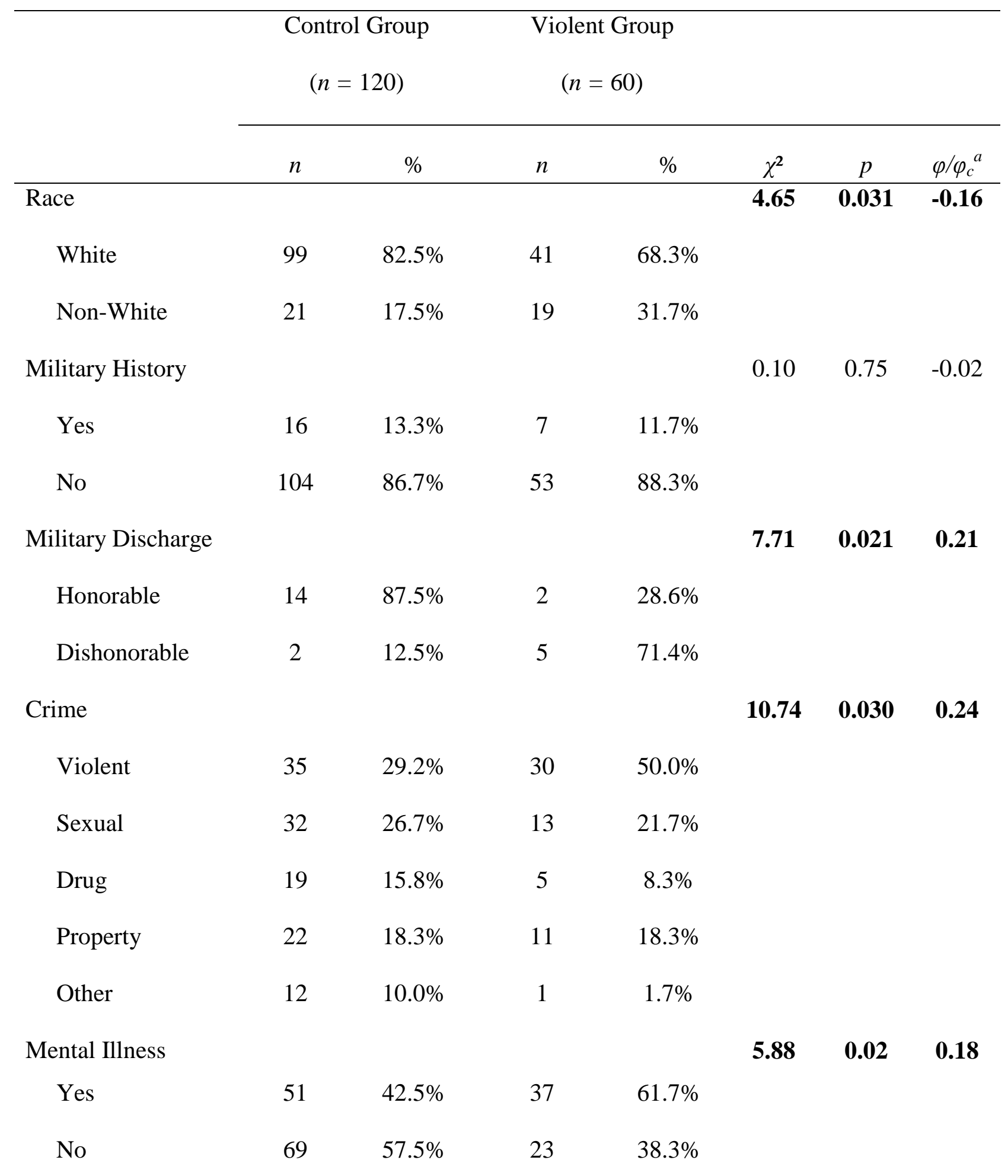


(Table 3 Continued)

MMPI-2-RF Given

$\begin{array}{lll}10.29 & 0.001 & -0.24\end{array}$

Yes

$120 \quad 100.0 \%$

55

$91.7 \%$

No, Refused

0

$0.0 \%$

5

$8.3 \%$

MMPI-2-RF Valid
7.89
$0.010-0.22$

Yes

114

$95.0 \%$

45

$81.8 \%$

No

6

$5.0 \%$

10

$18.2 \%$

Note. Statistically significant differences between the two groups are in bold.

${ }^{a} \varphi(\mathrm{Phi})=$ Used to measure effect size of categorical variables with two levels; $\varphi_{c}($ Cramer's V)

$=$ Used to measure effect size of categorical variables with more than two levels.

$\varphi / \varphi_{c}$ effect sizes: small $=0.10$, medium $=0.30$, large $=0.50$. 
Table 4

Continuous Demographic, Criminal, and Psychological Variables

\begin{tabular}{|c|c|c|c|c|c|c|c|}
\hline & \multicolumn{2}{|c|}{$\begin{array}{l}\text { Control Group } \\
\qquad(n=120)\end{array}$} & \multicolumn{2}{|c|}{$\begin{array}{l}\text { Violent Group } \\
\qquad(n=60)\end{array}$} & \multirow[b]{2}{*}{$t$} & \multirow[b]{2}{*}{$p$} & \multirow[b]{2}{*}{$\begin{array}{c}\text { Cohen's } \\
d\end{array}$} \\
\hline & $M$ & $S D$ & $M$ & $S D$ & & & \\
\hline Age at Incarceration (years) & 32.62 & 10.54 & 27.40 & 9.08 & -3.27 & 0.01 & 0.53 \\
\hline Sentence Length (years) & 6.15 & 6.50 & 8.79 & 9.51 & 2.19 & 0.03 & -0.32 \\
\hline VSI Scores & 0.16 & 0.52 & 3.38 & 2.15 & 15.60 & 0.001 & -2.06 \\
\hline Education (years) & 10.45 & 1.96 & 10.05 & 1.98 & -1.25 & 0.21 & 0.20 \\
\hline Beta-III IQ Score & 94.14 & 12.55 & 92.12 & 16.63 & -0.83 & 0.41 & 0.14 \\
\hline TCU Drug Screen II Scores & 2.95 & 3.20 & 3.64 & 3.52 & 1.30 & 0.20 & -0.21 \\
\hline MMPI-2-RF Scales & \multicolumn{2}{|c|}{$(n=120)$} & $\begin{array}{l}\text { Violent } \\
\qquad(n=\end{array}$ & $\begin{array}{l}\text { Group } \\
55)\end{array}$ & & & \\
\hline Cognitive Complaints & 51.39 & 13.10 & 53.65 & 14.53 & $\begin{array}{c}1.03 \\
F\end{array}$ & $\begin{array}{c}0.31 \\
p\end{array}$ & $\begin{array}{c}-0.16 \\
\text { Partial }^{\mathrm{b}} \\
\eta^{2}\end{array}$ \\
\hline Behavioral Externalization & 64.05 & 11.91 & 66.64 & 13.00 & 1.68 & 0.20 & 0.01 \\
\hline Antisocial & 65.76 & 12.33 & 69.45 & 13.32 & 3.22 & 0.07 & 0.02 \\
\hline Anger Proneness & 49.33 & 10.64 & 51.73 & 11.75 & 1.80 & 0.18 & 0.01 \\
\hline Aggression & 53.02 & 12.47 & 55.09 & 15.29 & 0.90 & 0.34 & 0.01 \\
\hline Aggressiveness-Revised & 54.71 & 10.02 & 57.53 & 11.95 & 2.64 & 0.11 & 0.02 \\
\hline
\end{tabular}

Note. VSI= Violence Severity Index Score; TCU = Texas Christian University; MMPI-2-RF = Minnesota Multiphasic Personality Inventory-2-Restructured Form.

${ }^{a}$ Cohen's $d$ effect sizes: small $=0.2$, medium $=0.5$, large $=0.8$.

${ }^{\mathrm{b}}$ Partial $\eta^{2}$ effect sizes: small $=0.01$, medium $=0.06$, large $=0.14$. 
Table 5

ANOVA for the MMPI-2-RF Personality Psychopathology Scales

\begin{tabular}{|c|c|c|c|c|c|c|c|}
\hline & \multicolumn{2}{|c|}{$\begin{array}{l}\text { Control Group } \\
\qquad(n=120)\end{array}$} & \multicolumn{2}{|c|}{$\begin{array}{l}\text { Violent Group } \\
\qquad(n=55)\end{array}$} & \multirow[b]{2}{*}{$F$} & \multirow[b]{2}{*}{$p$} & \multirow[b]{2}{*}{$\begin{array}{c}\text { Partial } \\
\eta^{2}\end{array}$} \\
\hline & $M$ & $S D$ & $M$ & $S D$ & & & \\
\hline Aggressiveness & 54.71 & 10.02 & 57.53 & 11.95 & 2.64 & 0.11 & 0.02 \\
\hline Disconstraint & 64.15 & 10.99 & 66.44 & 12.60 & 1.49 & 0.23 & 0.01 \\
\hline Introversion/Low Emotionality & 50.33 & 10.70 & 51.33 & 12.81 & 0.29 & 0.59 & 0.00 \\
\hline Neg. Emotion/Neuroticism & 47.75 & 9.17 & 49.64 & 12.08 & 1.30 & 0.26 & 0.01 \\
\hline Psychoticism & 48.35 & 11.26 & 54.78 & 18.10 & 8.24 & $0.005 *$ & $0.05^{\mathrm{a}}$ \\
\hline
\end{tabular}

Note. Neg. Emotion/Neuroticism = Negative Emotionality/Neuroticism scale; Introversion/Low Emotionality $=$ Introversion/Low Positive Emotionality scale.

$*$ Significance with Bonferroni correction, $p=.01$.

${ }^{a}$ Partial $\eta^{2}$ effect sizes: small $=0.01$, medium $=0.06$, large $=0.14$. 
Table 6

Bivariate correlations of regression variables

\begin{tabular}{lcccccc}
\hline & 1. & 2. & 3. & 4. & 5. & 6. \\
\hline 1. Age & - & -.052 & -.065 & .095 & -.025 & -.047 \\
2. Race & & - & $-.204 * *$ & .075 & .122 & $.191^{*}$ \\
3. Crime & & - & $-.428^{* *}$ & -.048 & .009 \\
4. Sentence Length & & & - & .135 & -.096 \\
5. Mental Health Hx & & & & - & -.122 \\
6. Psychoticism & & & & & -
\end{tabular}

Note. $\mathrm{Hx}=$ History. Psychoticism as measured on the MMPI-2-RF.

$* p<.05, * * p<.01$. 
Table 7

Binary Logistic Regression Predicting Violent and Control Group Inmates

\section{5\% Confidence \\ Interval for \\ $\operatorname{Exp}(\beta)$}

\begin{tabular}{|c|c|c|c|c|c|c|c|c|c|}
\hline & $\beta$ & $S E$ & Wald & $d f$ & Sig. & $\operatorname{Exp}(\beta)$ & Lower & Upper & $\begin{array}{c}\text { Correctly } \\
\text { Classified (\%) }\end{array}$ \\
\hline Block 1 & & & & & & & & & $67.6 \%$ \\
\hline Age & -0.07 & 0.02 & 9.03 & 1 & 0.00 & 0.93 & 0.89 & 0.98 & \\
\hline Race & 0.77 & 0.49 & 2.48 & 1 & 0.12 & 2.16 & 0.83 & 5.63 & \\
\hline Block 2 & & & & & & & & & $72.3 \%$ \\
\hline \multicolumn{10}{|l|}{ Crime } \\
\hline Violent & -0.01 & 0.49 & 0.00 & 1 & 0.98 & 0.99 & 0.38 & 2.58 & \\
\hline Sexual & -1.02 & 0.80 & 1.63 & 1 & 0.20 & 0.36 & 0.08 & 1.73 & \\
\hline Drug & -0.16 & 0.56 & 0.08 & 1 & 0.78 & 0.86 & 0.28 & 2.59 & \\
\hline Property & -2.21 & 1.13 & 3.82 & 1 & 0.05 & 0.11 & 0.01 & 1.01 & \\
\hline Sentence & 0.05 & 0.03 & 3.76 & 1 & 0.05 & 1.05 & 1.00 & 1.11 & \\
\hline Block 3 & & & & & & & & & $73.4 \%$ \\
\hline MH Hx & 0.98 & 0.39 & 6.32 & 1 & 0.01 & 2.68 & 1.24 & 5.76 & \\
\hline PSYC-r & 0.03 & 0.01 & 5.66 & 1 & 0.01 & 1.03 & 1.01 & 1.06 & \\
\hline
\end{tabular}

Note. Criterion variables were specified as $0=\operatorname{Control}, 1=$ Violent. $\operatorname{Exp}(\beta)$ is the odds ratio value. $\mathrm{MH} \mathrm{Hx}=$ Mental health history. PSYC-r = Psychoticism-Revised scale on the MMPI-2RF. 
Table 8

Sensitivity and Specificity of Logistic Regression Blocks

\begin{tabular}{|c|c|c|c|}
\hline & Control & Violent & Percentage Correct \\
\hline \multicolumn{4}{|l|}{ Block 1} \\
\hline Control & 109 & 9 & 92.4 \\
\hline Violent & 47 & 8 & 14.5 \\
\hline Overall Percentage & & & 67.6 \\
\hline \multicolumn{4}{|l|}{ Block 2} \\
\hline Control & 108 & 10 & 91.5 \\
\hline Violent & 38 & 17 & 30.9 \\
\hline Overall Percentage & & & 72.3 \\
\hline \multicolumn{4}{|l|}{ Block 3} \\
\hline Control & 105 & 13 & 89.0 \\
\hline Violent & 33 & 22 & 40.0 \\
\hline Overall Percentage & & & 73.4 \\
\hline
\end{tabular}


Table 9

Hierarchical Multiple Regression to Predict VSI scores

\begin{tabular}{|c|c|c|c|c|c|c|}
\hline \multirow[b]{2}{*}{ Predictors } & & \multicolumn{2}{|c|}{ Variables } & $\begin{array}{l}\text { + Crime } \\
\text { les }\end{array}$ & \multicolumn{2}{|c|}{$\begin{array}{c}+ \text { Psychological } \\
\text { Variables }\end{array}$} \\
\hline & $\beta$ & t value & $\beta$ & $\mathrm{t}$ value & $\beta$ & $\mathrm{t}$ value \\
\hline Age & -0.29 & $-3.98 *$ & -0.32 & $-4.57 *$ & -0.32 & $-4.68 *$ \\
\hline Race & 0.19 & $2.71 *$ & 0.16 & $2.23 * *$ & 0.17 & $2.38 * *$ \\
\hline Crime & & & -0.14 & -1.81 & -0.13 & -1.73 \\
\hline Sentence & & & 0.19 & $2.41 * *$ & 0.22 & $2.83^{*}$ \\
\hline Mental Health Hx & & & & & -0.18 & $-2.51 * *$ \\
\hline Psychoticism & & & & & 0.04 & 0.54 \\
\hline$R^{2}$ & & & & & & \\
\hline$\Delta R^{2}$ & & & & & & \\
\hline F value & & & & & & \\
\hline
\end{tabular}

Note. VSI = Violence Severity Index; Mental Health $\mathrm{Hx}=$ Mental Health History. $* p<.05, * * p<.01$. 
Table 10

Univariate Results for Cluster Differences on the MMPI-2-RF Personality Psychopathology Scales

\begin{tabular}{|c|c|c|c|c|c|c|c|c|c|}
\hline & \multicolumn{2}{|c|}{$\begin{array}{l}\text { Cluster } 1 \\
(n=11)\end{array}$} & \multicolumn{2}{|c|}{$\begin{array}{l}\text { Cluster } 2 \\
(n=15)\end{array}$} & \multicolumn{2}{|c|}{$\begin{array}{l}\text { Cluster } 3 \\
(n=29)\end{array}$} & \multirow[b]{2}{*}{$F$} & \multirow[b]{2}{*}{$P$} & \multirow[b]{2}{*}{$\begin{array}{c}\text { Partial } \\
\eta^{2}\end{array}$} \\
\hline & $M$ & $S D$ & $M$ & $S D$ & $M$ & $S D$ & & & \\
\hline Aggressiveness & $53.45_{\mathrm{a}}$ & 11.15 & $72.00_{\mathrm{a}, \mathrm{b}}$ & 7.50 & $51.59_{\mathrm{b}}$ & 6.92 & 33.55 & $0.00 *$ & 0.56 \\
\hline Psychoticism & $78.64_{a, b}$ & 17.65 & $59.53 \mathrm{a}, \mathrm{c}$ & 14.87 & $43.28_{b, c}$ & 6.07 & 37.46 & $0.00 *$ & 0.59 \\
\hline Disconstraint & 68.45 & 13.51 & $76.07 \mathrm{a}$ & 8.09 & $60.69 \mathrm{a}$ & 11.08 & 10.67 & $0.00 *$ & 0.28 \\
\hline Neg. Emotion/Neuroticism & $67.64_{a, b}$ & 10.70 & $49.87 \mathrm{a}, \mathrm{c}$ & 7.43 & $42.69_{b, c}$ & 5.98 & 44.20 & $0.00 *$ & 0.63 \\
\hline Introversion/Low Emotion & 58.55 & 12.56 & 45.60 & 9.02 & 51.55 & 13.47 & 3.56 & 0.04 & 0.12 \\
\hline
\end{tabular}

Note. Neg. Emotion/Neuroticism = Negative Emotionality/Neuroticism scale. Introversion/Low Emotion $=$ Introversion/Low Positive Emotionality scale. The means and standard deviation values were calculated based on scale values and not converted z-scores, which were used for the cluster analysis.

a, b, c Means in the same row with the same subscript are significantly different from one another based upon Tukey's post-hoc analyses.

$* p<.001$. Bonferroni corrections $p=.01$.

$* *$ Partial $\eta^{2}$ effect sizes: small $=0.01$, medium $=0.06$, large $=0.14$. 
Table 11

Categorical Demographic, Criminal, and Psychological Variables for Violent Inmate Clusters

\begin{tabular}{|c|c|c|c|c|c|c|c|c|c|}
\hline & \multicolumn{2}{|c|}{$\begin{array}{l}\text { Cluster 1 } \\
(n=11)\end{array}$} & \multicolumn{2}{|c|}{$\begin{array}{l}\text { Cluster } 2 \\
(n=15)\end{array}$} & \multicolumn{2}{|c|}{$\begin{array}{l}\text { Cluster } 3 \\
(n=29)\end{array}$} & \multirow[b]{2}{*}{$\chi^{2}$} & \multirow[b]{2}{*}{$p$} & \multirow[b]{2}{*}{$\varphi / \varphi_{c}{ }^{a}$} \\
\hline & $n$ & $\%$ & $n$ & $\%$ & $n$ & $\%$ & & & \\
\hline Race & & & & & & & 1.24 & 0.54 & 0.15 \\
\hline White & 8 & 72.7 & 9 & 60.0 & 22 & 75.9 & & & \\
\hline Non-White & 3 & 27.3 & 6 & 40.0 & 7 & 24.1 & & & \\
\hline History of Special Ed & & & & & & & 6.36 & .042 & 0.35 \\
\hline Yes & 8 & 72.7 & 6 & 40.0 & 8 & 27.6 & & & \\
\hline No & 3 & 27.3 & 8 & 53.3 & 20 & 69.0 & & & \\
\hline Military History & & & & & & & 2.63 & 0.27 & 0.22 \\
\hline Yes & 0 & 0.0 & 3 & 20.0 & 3 & 10.3 & & & \\
\hline No & 11 & 100.0 & 12 & 80.0 & 26 & 89.7 & & & \\
\hline Military Discharge & & & & & & & 4.30 & 0.37 & 0.20 \\
\hline Honorable & N/A & N/A & 0 & 0.0 & 1 & 33.3 & & & \\
\hline Dishonorable & N/A & N/A & 3 & 100.0 & 2 & 66.7 & & & \\
\hline Conviction Crime & & & & & & & 15.34 & 0.05 & 0.37 \\
\hline Violent & 4 & 36.4 & 7 & 46.7 & 16 & 55.2 & & & \\
\hline Sexual & 5 & 45.5 & 1 & 6.7 & 7 & 24.1 & & & \\
\hline Drug & 0 & 0.0 & 3 & 20.0 & 0 & 0.0 & & & \\
\hline Property & 2 & 18.2 & 3 & 20.0 & 6 & 20.7 & & & \\
\hline Other & 0 & 0.0 & 1 & 6.7 & 0 & 0.0 & & & \\
\hline
\end{tabular}


(Table 11 Continued)

Mental Health History

$2.74 \quad 0.25 \quad 0.22$

Yes

$\begin{array}{llllll}9 & 81.8 & 8 & 53.3 & 16 & 52.2\end{array}$

No

$\begin{array}{llllll}2 & 18.2 & 7 & 46.7 & 13 & 44.8\end{array}$

VSI Scores

$22.26 \quad 0.14 \quad 0.45$

$\begin{array}{lllllll}1 & 6 & 54.5 & 1 & 6.7 & 5 & 17.2 \\ 2 & 0 & 0.0 & 5 & 33.3 & 2 & 6.9 \\ 3 & 2 & 18.2 & 4 & 26.7 & 10 & 34.5 \\ 4 & 3 & 27.3 & 2 & 13.3 & 6 & 20.7 \\ 5 & 0 & 0.0 & 1 & 6.7 & 3 & 10.3 \\ 6 & 0 & 0.0 & 0 & 0.0 & 1 & 3.4 \\ 7 & 0 & 0.0 & 1 & 6.7 & 0 & 0.0 \\ 8 & 0 & 0.0 & 1 & 6.7 & 1 & 3.4 \\ 9 & 0 & 0.0 & 0 & 0.0 & 0 & 0.0 \\ 10 & 0 & 0.0 & 0 & 0.0 & 1 & 3.4\end{array}$

Note. Statistically significant differences between the two groups are in bold.

${ }^{a} \varphi(\mathrm{Phi})=$ Used to measure effect size of categorical variables with two levels; $\varphi_{c}$ (Cramer's V)

$=$ Used to measure effect size of categorical variables with more than two levels. $\varphi / \varphi_{c}$ effect sizes: small $=0.10$, medium $=0.30$, large $=0.50$. 
Table 12

Continuous Demographic, Criminal, and Psychological Variables for Violent Inmate Clusters

\begin{tabular}{|c|c|c|c|c|c|c|c|c|c|}
\hline & \multicolumn{2}{|c|}{$\begin{array}{l}\text { Cluster } 1 \\
(n=11)\end{array}$} & \multicolumn{2}{|c|}{$\begin{array}{l}\text { Cluster } 2 \\
(n=15)\end{array}$} & \multicolumn{2}{|c|}{$\begin{array}{l}\text { Cluster } 3 \\
(n=29)\end{array}$} & \multirow[b]{2}{*}{$F$} & \multirow[b]{2}{*}{$p$} & \multirow[b]{2}{*}{$\begin{array}{c}\text { Partial } \\
\eta^{2}\end{array}$} \\
\hline & $M$ & $S D$ & $M$ & $S D$ & $M$ & $S D$ & & & \\
\hline Age at Incarceration (yrs) & $31.00^{\mathrm{a}}$ & 11.31 & $23.20^{\mathrm{a}}$ & 4.41 & 27.79 & 8.36 & 3.03 & 0.05 & .10 \\
\hline Age at Violent Act (yrs) & 31.30 & 9.75 & 25.67 & 4.48 & 30.79 & 10.71 & 1.77 & 0.18 & .07 \\
\hline Years of Education & 9.00 & 1.79 & 10.67 & 1.45 & 10.10 & 2.21 & 2.35 & 0.11 & .08 \\
\hline Length of Sentence (yrs) & 6.18 & 4.90 & 5.30 & 5.77 & 11.69 & 11.78 & 2.84 & 0.07 & .10 \\
\hline Time in Prison (months) & 76.45 & 58.01 & 78.53 & 53.99 & 106.44 & 92.47 & 0.94 & .396 & .04 \\
\hline Beta-III IQ Score & $81.00^{\mathrm{a}}$ & 21.41 & 90.20 & 10.41 & $96.90^{\mathrm{a}}$ & 17.05 & 3.62 & 0.03 & .12 \\
\hline TCU Drug Screen II Score & 3.64 & 2.84 & 5.14 & 3.96 & 2.96 & 3.44 & 1.84 & 0.17 & .07 \\
\hline VSI Scores & 2.18 & 1.40 & 3.40 & 1.96 & 3.52 & 2.03 & 2.06 & 0.14 & .07 \\
\hline
\end{tabular}

Note. VSI = Violence Severity Index. Partial $\eta^{2}$ effect sizes: small $=0.01$, medium $=0.06$, large $=0.14$.

${ }^{a}$ Means in the same row with the same subscript are significantly different from one another. 
Figure 1

MMPI-2-RF Personality Scales for Violent Inmate Clusters

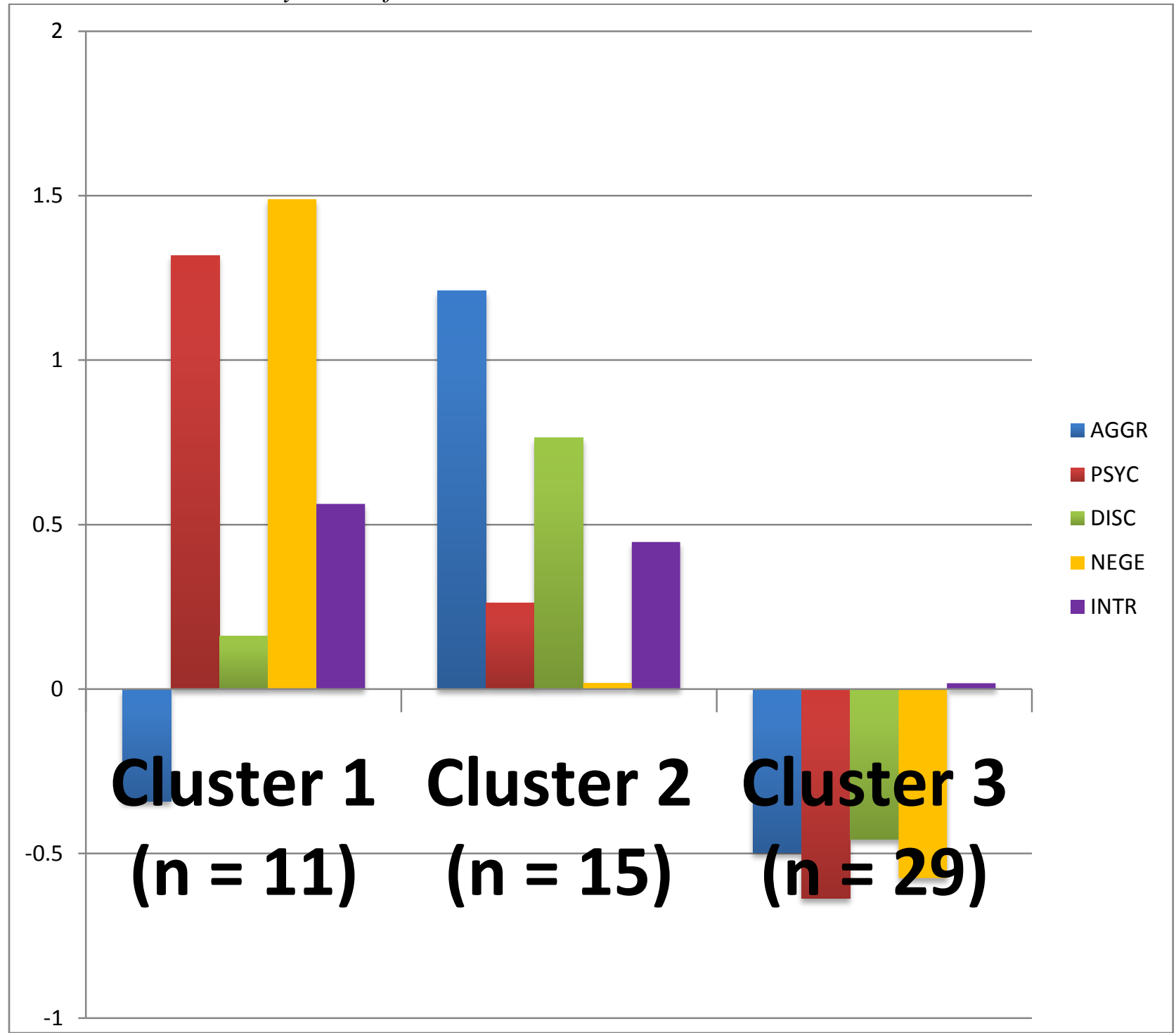

Note. These values are scores converted to z-scores. AGGR = Aggressiveness; PSYC = Psychoticism; DISC $=$ Disconstraint; NEGE = Negative Emotionality $/$ Neuroticism; INTR $=$ Low Positive Emotionality/Introversion. 
Appendix A

\section{TCU DRUG SCREEN II}

During the last 12 months (before being locked up, if applicable) -

1. Did you use larger amounts of drugs or use them for a longer time than you planned or intended?

2. Did you try to cut down on your drug use but were unable to do it?

3. Did you spend a lot of time getting drugs, using them, or recovering from their use?

4a. Did you get so high or sick from using drugs that it kept you from doing work, going to school, or caring for children?

4b. Did you get so high or sick from drugs that it caused an accident or put you or others in danger?

5. Did you spend less time at work, school, or with friends so that you could use drugs?

6a. Did your drug use cause emotional or psychological problems?

6b. Did your drug use cause problems with family, friends, work, or police?

6c. Did your drug use cause physical health or medical problems?

7. Did you increase the amount of a drug you were taking so that you could get the same effects as before?

8. Did you ever keep taking a drug to avoid withdrawal symptoms or keep from getting sick?

9. Did you get sick or have withdrawal symptoms when you quit or missed taking a drug?

10. Which drug caused the most serious problem? [CHOOSE ONE]
None
O Alcohol
O Marijuana/Hashish
O Hallucinogens/LSD/PCP/Psychedelics/Mushrooms
O Inhalants
O Crack/Freebase
$\bigcirc$ Heroin and Cocaine (mixed together as Speedball)
O Cocaine (by itself)
O Heroin (by itself)
O Street methadone (non-prescription)
O Other Opiates/Opium/Morphine/Demerol
O Methamphetamines
O Amphetamines (other uppers)
O Tranquilizers/Barbiturates/Sedatives (downers) 


\begin{tabular}{|c|c|c|c|c|c|}
\hline $\begin{array}{l}\text { How often did you use each type of drug } \\
\text { during the last } 12 \text { months? }\end{array}$ & Never & $\begin{array}{l}\text { Only } \\
\text { a few } \\
\text { times }\end{array}$ & $\begin{array}{c}1-3 \\
\text { times per } \\
\text { month }\end{array}$ & $\begin{array}{c}1-5 \\
\text { times per } \\
\text { week }\end{array}$ & $\begin{array}{c}\text { About } \\
\text { every } \\
\text { day }\end{array}$ \\
\hline 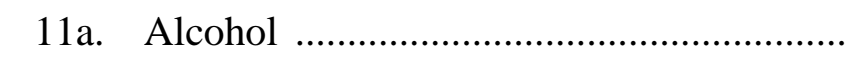 & 0 & 0 & 0 & 0 & 0 \\
\hline 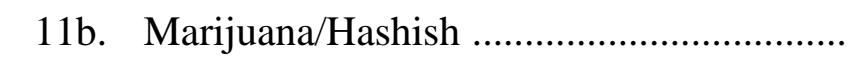 & O & O & O & O & O \\
\hline 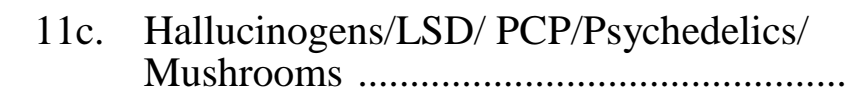 & 0 & 0 & 0 & 0 & 0 \\
\hline 11d. Inhalants ....................... & O & O & O & O & O \\
\hline 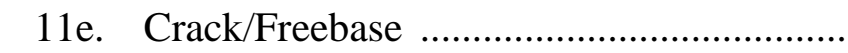 & 0 & 0 & 0 & 0 & 0 \\
\hline $\begin{array}{l}\text { 11f. } \begin{array}{l}\text { Heroin and Cocaine } \\
\text { (mixed together as Speedball) }\end{array}\end{array}$ & 0 & 0 & 0 & 0 & 0 \\
\hline 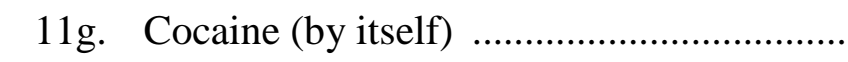 & O & O & O & O & O \\
\hline 11h. Heroin (by itself) & 0 & 0 & 0 & 0 & 0 \\
\hline 11i. Street Methadone (non-prescription) .......... & O & O & O & O & O \\
\hline $\begin{array}{ll}\text { 11j. } & \text { Other Opiates/Opium/Morphine/ } \\
\text { Demerol }\end{array}$ & O & O & O & O & O \\
\hline 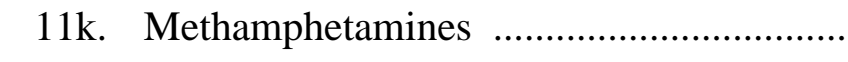 & O & O & O & O & O \\
\hline 111. Amphetamines (other uppers) ........................ & O & O & O & O & O \\
\hline 11m. Tranquilizers/Barbiturates/Sedatives & O & O & 0 & O & 0 \\
\hline 11n. Other (specify) & 0 & 0 & 0 & 0 & 0 \\
\hline
\end{tabular}

12. During the last 12 months, how often did you inject drugs with a needle?

O Never O Only afew times O 1-3 times/month O 1-5 times per week O Daily

13. How serious do you think your drug problems are?

O Notatall O Slightly O Moderately O Considerably O Extremely

14. How many times before now have you ever been in a drug treatment program? [DO NOT INCLUDE AA/NA/CA MEETINGS]
O Never
O 1 time
O 2 times
O 3 times
O 4 or more times

15. How important is it for you to get drug treatment now?

O Not at all O Slightly O Moderately O Considerably O Extremely


Appendix $B$

$\underline{\text { Data Collection Form }}$

Initials:

DOC \#:

DEMOGRAPHIC VARIABLES

Date of Birth:

Race:

White

Non-White

Years of Education:

\begin{tabular}{llll} 
Circle One: & $\begin{array}{l}\text { Graduated H.S. } \\
\text { Graduated College }\end{array}$ & GED & Other: \\
\hline
\end{tabular}

History of Special Education? $\quad$ Yes No

Occupation:

Marital Status: $\quad$ Single $\quad$ Married Divorced Widowed Separated

Military History: $\quad$ Yes No

If yes, what branch of the military did the inmate serve in?

If yes, honorable discharge: $\quad$ Yes No

\section{CRIMINAL HISTORY VARIABLES}

Current Instant Offense:

Sentence Length:

Secondary Offense:

Sentence Length:

Other Offenses:

Sentence Length(s):

If multiple sentences, are the sentences ran: Concurrent or Consecutive 
Parole Eligible? $\quad$ Yes No

Parole Eligibility Date:

Minimum Discharge Date:

Effective Incarceration Date:

Documented Gang Affiliation?

Yes No

If yes, what gang does the inmate claim?

$\begin{array}{lll}\text { Prior convictions? } & \text { Yes } & \text { No }\end{array}$

Number of prior convictions?

Prior Prison Sentence? $\quad$ Yes No

If yes, length of time served (in months):

$\begin{array}{llll}\text { Circle all that apply: } & \text { State Prison } & \text { Federal } & \text { Jail Time }\end{array}$

$\begin{array}{llllll}\text { Current Custody Level: } & \text { I } & \text { II } & \text { III } & \text { IV } & \text { V }\end{array}$

(I= community; II = minimum; $\mathrm{III}=$ medium; $\mathrm{IV}=$ close $; \mathrm{V}=$ maximum $)$

Prison Classes/Programming: Passed

Removed

Refused

Failed

Withdrawn

PSYCHOLOGICAL VARIABLES

Documented Mental Health History? Yes No

If yes, diagnosis:

Current psychotropic medications:

Yes No

If yes, what is the inmate prescribed?

DOC Psychological Stability Code (circle one):

History of Suicide Attempts? Yes

$\begin{array}{lllll}1 & 2 & 3 & 4 & 5\end{array}$

If yes, when?

Was the attempt: in the community or while incarcerated 
MMPI-2-RF

MMPI-2-RF Administered: Yes

No If no, why $?$

Valid Profile:

Yes

No

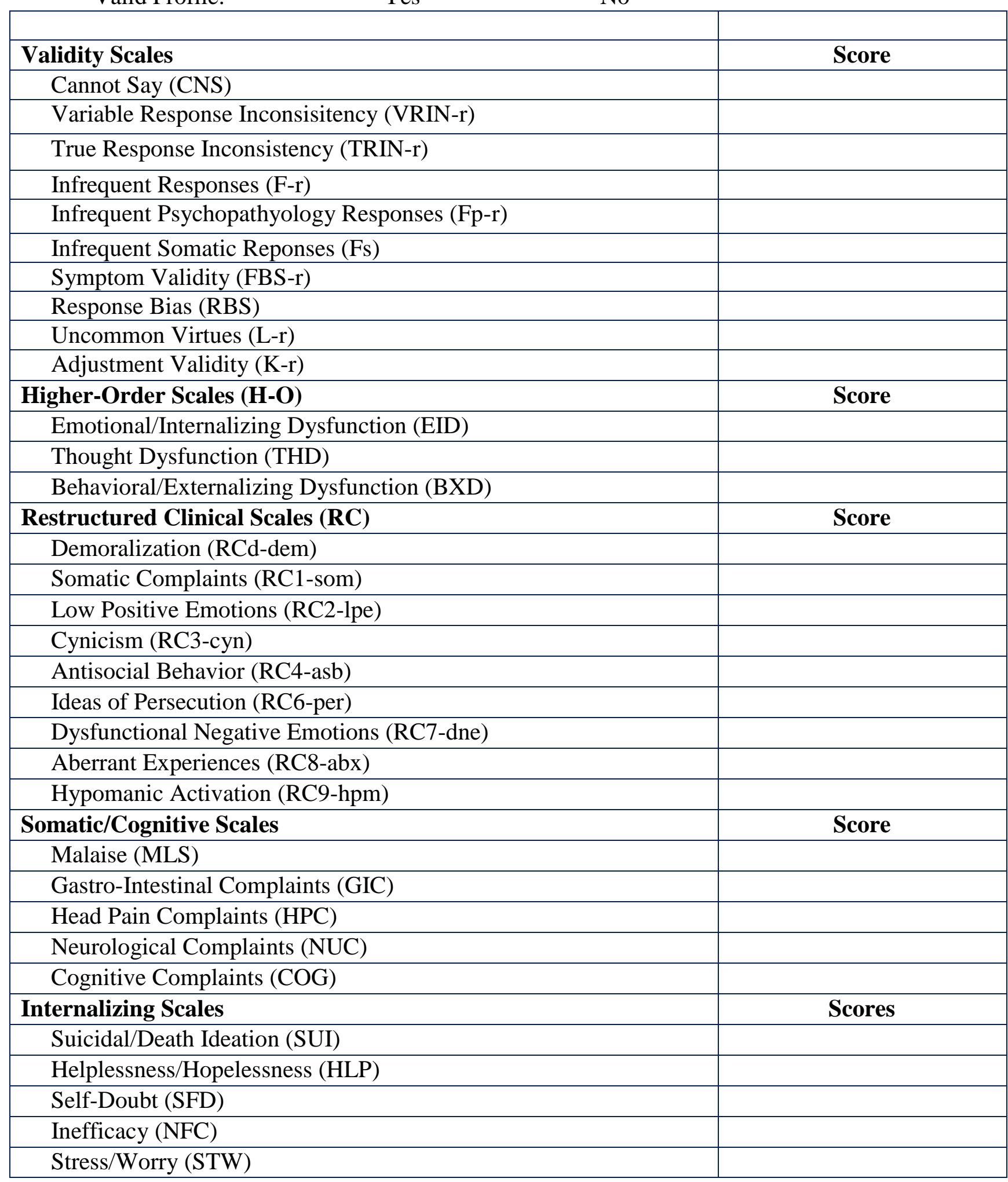




\begin{tabular}{|l|l|}
\hline Anxiety (AXY) & \\
\hline Anger Proneness (ANP) & \\
\hline Behavior-Restricting Fears (BRF) & \\
\hline Multiple Specific Fears (MSF) & Score \\
\hline Externalizing Scales & \\
\hline Juvenile Conduct Problems (JCP) & \\
\hline Substance Abuse (SUB) & \\
\hline Aggression (AGG) & \\
\hline Activation (ACT) & Score \\
\hline Interpersonal Scales & \\
\hline Family Problems (FML) & \\
\hline Interpersonal Passivity (IPP) & \\
\hline Social Avoidance (SAV) & \\
\hline Shyness (SHY) & Score \\
\hline Disaffiliativeness (DSF) & \\
\hline Interest Scales & \\
\hline Aesthetic-Literary Interests (AES) & Score \\
\hline Mechanical-Physical Interests (MEC) & \\
\hline PSY-5 (Personality Psychopathology Five) Scales Revised & \\
\hline Aggressiveness-Revised (AGGR-r) & \\
\hline Psychoticism-Revised (PSYC-r) & \\
\hline Disconstraint-Revised (DISC-r) & \\
\hline Negative Emotionality/Neuroticism-Revised (NEGE-r) & \\
\hline Introversion/Low Positive Emotionality-Revised (INTR-r) & \\
\hline
\end{tabular}

\section{TCU Drug Screen II}

\section{Total Score}

Substances endorsed:

\section{BETA-III}

\begin{tabular}{|l|l|l|}
\hline BETA-III Subtests & Score & Percentile Rank \\
\hline Coding & & \\
\hline Picture Completion & & \\
\hline Clerical Checking & & \\
\hline Picture Absurdities & & \\
\hline Matrix Reasoning & & \\
\hline Total Beta-III Score & & \\
\hline
\end{tabular}

\section{Trails Making Test (TMT)}

$\begin{array}{lllll}\mathbf{A}= & \text { Perfectly Normal } & \text { Normal } & \text { Mild/Mod } & \text { Mod/Severely Impaired } \\ \mathbf{B}= & \text { Perfectly Normal } & \text { Normal } & \text { Mild/Mod } & \text { Mod/Severely Impaired }\end{array}$




\section{INSTITUTIONAL BEHAVIOR}

Is this inmate's file flagged for a classification category? $\quad$ Yes No

If yes, what is the classification group (e.g., assaultive, suicide risk):

How many GUILTY institutional misconduct write-ups does the inmate have?

Does the inmate have any write-ups for violent behavior? Yes No Potentially

How many write-ups for violent behavior while incarcerated does the inmate have?

Answer the following questions for each documented incident of violent behavior:

When did the incident occur (month/date)?

What was the write-up classified as (e.g., assault, fighting)?

Was the victim: an inmate or staff member

Was a weapon used? $\quad$ Yes No

If yes, what type of weapon?

Was anyone injured?

Yes

No

If yes, describe the injury.

What level of treatment was needed? On-Site or Off-site

Circle one: First-aid Stiches Casting/Sprain/Sling Hospitalization Emergency Medical Care

What was the disposition and sanctions of the write-up?

Does the inmate have any write-ups for any of the following acts:

$\begin{array}{lccc}\text { Possession of a Weapon: } & \text { Yes } & \text { No } & \text { How many? } \\ \text { Verbal Threats: } & \text { Yes } & \text { No } & \text { How many? } \\ \text { Creating a Disturbance: } & \text { Yes } & \text { No } & \text { How many? } \\ \text { Fighting: } & \text { Yes } & \text { No } & \text { How many? }\end{array}$




\section{Allison M. Schenk, M.S. \\ 3660 South Cox Avenue Apartment \#1409 \\ Springfield, MO 65807}

(414) 331 - 3721

Allison.M.Schenk@gmail.com

\section{EDUCATION}

University of Wisconsin-Madison

Bachelor of Arts: Psychology May 2009

Certificate in Criminal Justice May 2009

Graduated with Honors from the School of Letters and Sciences

GPA: $3.8 / 4.0$

West Virginia University

Master of Science: Psychology

Certificate in University Teaching

Doctorate of Philosophy: Psychology

Dissertation: Psychological Measures to

Predict Serious Prison Violence
May 2011

May 2013

August 2014 (Anticipated

Graduation)

Defended March $31^{\text {st }}, 2014$

GPA: $4.0 / 4.0$

\section{CLINICAL EXPERIENCE}

Predoctoral Psychology Intern

\section{United States Medical Center for Federal Prisoners, Springfield, MO}

40 hours/week

Internship Training Director: Elizabeth Tyner, Ph.D.

Provide for the mental health needs of pre-trial, civilly committed, and sentenced male inmates at a federal medical center housing all security levels.

- Complete forensic evaluations (e.g., competency to proceed, criminal responsibility) involving multiple clinical interviews, psychological testing, and behavioral observations of defendants

- Conduct diagnostic and observation evaluations of sentenced inmates to determine if involuntary hospitalization and treatment is needed

- Lead three weekly competency restoration groups of pre-trial defendants as part of an effort to restore to competency

- Respond in a timely and effective manner to crises including: disruptive behaviors, selfinjurious and suicidal inmates, involuntary administration of medications of Due Processed individuals, and other potentially dangerous situations that arise

- Attend weekly forensic seminars to present and discuss landmark decisions in mental health case law 
- Learn clinical skills and discuss clinically-relevant topics during weekly didactic trainings

- Work among an interdisciplinary team planning and implementing the treatment of severely mentally ill individuals

- Serve as an integral team member in risk assessment evaluations of civilly committed individuals and those found Not Guilty by Reason of Insanity (NGRI)

- Maintain a caseload of individual therapy inmates and lead weekly therapy groups on a variety of mental health topics throughout the year (e.g., anger management, coping with medical illnesses, dialectical behavior therapy)

- Serve the mental health needs of medically-referred inmates, complete organ transplant and interferon treatment evaluations, and conduct neuropsychological testing for inmates potentially suffering from conditions such as dementia, traumatic brain injury, and other neuropathological conditions

- Conduct psychoeducational and process groups in a residential drug abuse treatment setting within the institution

Graduate Student Clinical Psychology Intern

July 2012-June 2013 Federal Correctional Institute (FCI-Morgantown), Morgantown, WV

18 hours/week

Supervisor: Edward Baker, Ph.D.

Provided for the mental health needs of male inmates in a low-security federal prison camp.

- Screened incoming inmates for mental health needs and completed follow-up services, if necessary

- Administered personality, intelligence, and neuropsychological assessments of inmates

- Assisted with mental status examinations of inmates in the Special Housing Unit (SHU)

- Conducted individual therapy with inmates struggling with different mental health problems such as depression, anxiety, suicidal ideation, and life stressors (divorce, death of loved ones, re-entry)

- Assessed inmates on psychotropic medications and communicated with medical staff to coordinate care

- Evaluated the mental health histories of inmates to determine their eligibility for Interferon treatment

- Assessed inmates' history of substance abuse and conducted clinical interviews as part of the qualification process for a Residential Drug Abuse Program (RDAP)

\section{Graduate Student Therapist}

Fremouw-Sigley Psychological Associates, Morgantown, WV

August 2011-May 2013

8-10 hours/week

Supervisor: Terry Sigley, M.A.

Served as a therapist in an outpatient mental health clinic with child, adolescent, and adult clients.

- Conducted individual therapy with clients seeking mental health treatment for issues such as depression, anxiety, adjustment disorders (e.g., divorce, death), personality disorders, oppositional defiant disorder, and sexual/physical victimization history

- Led individual and group outpatient sex offender treatment with adjudicated juvenile males 
- Formed treatment plans with clients based upon empirically supported treatment methods for the presenting problem(s)

- Coordinated treatment with other agencies such as social workers, Child Protective Services, Department of Health and Human Resources, probation officers, lawyers, and schools

- Met bi-weekly for supervision with a licensed psychologist to discuss therapy cases, treatment techniques, and professional development issues

Graduate Student Clinical Psychology Intern West Virginia Industrial Home for Youth, Salem, WV

16 hours/week

Supervisor: Tom Horacek, Ph.D.
July 2011-June 2012

Addressed the mental health needs of juvenile offenders in a maximum-security detention facility (ages 11-21; males and females).

- Completed court-ordered diagnostic psychological evaluations for adjudicated juveniles, which included treatment and placement recommendations to best meet the youth's needs, before sentencing

- Interpreted and integrated psychological testing to support placement and treatment recommendations

- Communicated treatment needs and placement recommendations in a multidisciplinary team setting, which included judges, lawyers, social workers, probation officers, and parents

- Completed structured risk assessments of adjudicated juvenile sex offenders (e.g., JSOAPII, ERASOR)

- Administered semi-structured clinical interviews assessing traumatic experiences of youth (CAPS-CA)

- Conducted short-term individual and group therapy

Graduate Student Therapist Quin Curtis Center, Morgantown, WV

September 2011 - July 2012

4 hours/week

Supervisor: Daniel W. McNeil, Ph.D.

Offered therapy to clients, ranging in age from 21 to 65 years old, in a university-operated community mental health clinic.

- Conducted weekly therapy sessions with clients who had various anxiety disorders (e.g., hoarding, obsessive-compulsive disorder, generalized anxiety, specific phobias)

- Administered, scored, and provided feedback on psychological assessment measures

- Consulted with a team of colleagues and a licensed clinical psychologist on a weekly basis regarding patients, treatment planning, and ethical issues

Psychology Graduate Student Intern Pruntytown Correctional Center, Pruntytown, WV

July 2010 - June 2011

16 hours/week

Supervisor: Tom Horacek, Ph.D.

Served as a graduate student intern at a minimum/medium-security state prison. 
- Conducted intake evaluations for new inmates consisting of a thorough records review, clinical interviews, testing and interpretation, and mental status assessments written in an integrated report

- Updated psychological evaluation reports for inmates considered for parole or work release decisions

- Screened and assessed medication needs of inmates requesting psychotropic medications or to alter their medication regimen, and communicated this information to medical staff

- Facilitated monthly telecommunication appointments between inmates and the psychiatrist

- Responded to crisis situations such as suicide threats/attempts and inmate conflicts

- Conducted one-on-one therapy sessions with inmates seeking mental health services for various problems (e.g., anxiety, depression, anger management, traumatic events)

Graduate Student Therapist Quin Curtis Center, Morgantown, WV

September 2009 - May 2010

8 hours/week

Supervisor: Jennifer Myers, Ph.D.

Provided for the mental health needs of adolescent and adult clients in a university-operated community mental health clinic.

- Conducted weekly therapy sessions with clients seeking mental health services for externalizing (e.g., conduct disorder, anger management) and internalizing disorders (e.g., depression, social anxiety)

- Participated in co-therapy with a colleague

- Administered, scored, and provided feedback on psychological assessment measures

Clinical Assistant to a Forensic Psychologist August 2009 - July 2013 William J. Fremouw, Ph.D., ABPP, Clinical \& Forensic Psychologist, Morgantown, WV Monthly (average)

Supervisor: William J. Fremouw, Ph.D., ABPP

Actively participated and gained first-hand experience with the duties and responsibilities of a clinical forensic psychologist.

- Assisted with various forensic evaluations including: competency to stand trial, criminal responsibility, and dangerousness risk assessments

- Observed Dr. Fremouw testify as an expert witness in court

- Conducted portions of the clinical interviews and administered psychological and forensic-specific measures (e.g., MacCAT-CA, Georgia Court Competency Test, TOMM, SIRS-2 M-Fast, HCR-20)

\section{RESEARCH EXPERIENCE}

Graduate Student Researcher on Forensic Psychology Issues August 2009 - Present Department of Psychology, West Virginia University, Morgantown, WV

5 hours/week (average)

Supervisor: William Fremouw, Ph.D., ABPP 
Conduct research on a variety of forensically-relevant psychological topics.

- Engineered multiple research projects about different components of bullying and cyberbullying

- Contributed to the creation of a suicide risk assessment matrix for assessing suicide risk with clients

- Critically reviewed the existing literature and published a paper investigating the relation between inmate variables and prison violence

- Designed and implemented a records-review study to investigate how psychological variables relate to prison violence among inmates

- Presented forensically-relevant research nationally and internationally at AP-LS conferences

- Published seven journal articles, one book chapter, and one book review on forensic topics

Graduate Student Researcher on Juvenile Sex Offender Topics Sept. 2010 - July 2013 Chestnut Ridge Center, Morgantown, WV

Harriet B. Jones Treatment Center, Salem, WV

4 hours/week (average)

Supervisor: Christi Cooper-Lehki, D.O.

Engineered research focusing on characteristics of adjudicated juvenile sexual offenders and components of their treatment program.

- Discussed the characteristics and risk factors of juvenile sex offenders and how these variables relate to offending behaviors in terms of conceptualizing research projects

- Collected and analyzed data on the differences in information obtained from self-report measures versus polygraph assessments

- Prepared, edited, and submitted a report based on the discrepancy between polygraph and self-report information

Clinical Research Assistant

September 2008 - May 2009

Mendota Juvenile Treatment Center at Mendota Mental Health Institute, Madison, WI 10 hours /week

Supervisors: David McCormick, Ph.D. and Michael Caldwell, Psy.D.

Conducted research with a special population of juvenile offenders in a secure forensic unit to monitor changes in behavioral traits, such as aggression, anger, and callousness, in an effort to better understand which behavioral changes are the most influential on future conduct and reducing recidivism rates.

- Attended individual therapy sessions, juvenile offender review hearings, and collaborative case management meetings to receive direct exposure to the interaction of psychology and the juvenile justice system

- Scored and entered data gathered from evaluations completed by the youth upon admission and every subsequent 90-days, examining traits of aggression, antisocial personality, impulsivity, and indicators of psychopathic behaviors

- Constructed summaries from police reports and background information about the youth for clinicians to utilize in therapy sessions

- Scored dynamic risk factors for juvenile sex offenders based upon a thorough review of records 
Research Assistant

September 2007 - May 2009

University of Wisconsin Psychopathy Research Laboratory, Madison, WI

10 hours/week

Supervisor: Joseph P. Newman, Ph.D.

Served as a research assistant on a 5-year NIMH funded grant (5R01MH053041-11; PI:

Joseph P. Newman and John Curtin) that explored information processing deficits associated with psychopathy and other syndromes of disinhibition.

- Examined the effects of manipulating working memory load and the use of secondary emotion cues of shock to moderate fear potentiated startle responses in undergraduate students

- Ran a 2.5 hour EEG protocol, while measuring psychophysiological brain activity and startle responses as participants completed a working memory task

- Administered a computerized survey that measured a variety of individual difference variables, including anxiety, psychopathy, and reward sensitivity through the use of the Psychopathy Checklist-Revised (PCL-R) and Multidimensional Personality Questionnaire (MPQ)

\section{Research Assistant}

November 2005 - August 2009

\section{Center for Tobacco Research and Intervention, Madison and Milwaukee, WI}

12 hours/week

Supervisors: Douglas Jorenby, Ph.D. (Madison); Meg Feyen, M.S. (Milwaukee)

Investigated various smoking cessation methods at the University of Wisconsin-School of Medicine and Public Health's Center for Tobacco Research and Intervention's (CTRI) National Institute on Drug Abuse funded Transdisciplinary Tobacco Use Research Center (TTURC).

- Worked as an integral component of a three year study of smoking cessation in the Milwaukee and Madison areas to create an algorithm of the most effective smoking cessation methods by demographic characteristics

- Collaborated with team members to manage a case load of over 2,000 participants in multiple concurrent research studies

- Completed extensive telephone interviews and subsequent follow-up calls to obtain medical histories, assess medication use, side effects, mood (specifically symptoms of depression), smoking triggers, and environmental influences on smoking statuses

- Performed quality control on completed files to guarantee accuracy and completeness of data

\section{PUBLICATIONS}

Keelan, C. M., Schenk, A. M., McNally, M., \& Fremouw, W. J. (2014). The interpersonal worlds of bullies: Parents, peers, and partners. The Journal of Interpersonal Violence, 29(7), 1338-1353. 
Schenk, A. M., Cooper-Lehki, C., Keelan, C. M., \& Fremouw, W. J. (2014). Underreporting of bestiality among juvenile sex offenders: Polygraph versus self-report. Journal of Forensic Sciences, 59 (2), 540-542.

Schenk, A. M., Fremouw, W. J., \& Keelan, C. M. (2013). Characteristics of college cyberbullies. Computers in Human Behavior, 29 (6), 2320-2327.

Schenk, A. M. \& Fremouw, W. J. (2012). Individual characteristics related to prison violence: A critical review of the literature. Aggression and Violent Behavior, 17 (5), 430-442.

Schenk, A. M. \& Fremouw, W. (2012). Prevalence, psychological impact, and coping of cyberbully victims among college students. Journal of School Violence, 11 (1), 21-37.

Schenk, A. M., Ragatz, L., \& Fremouw, W. (2012). Vicious dogs part 2: Criminal thinking, callousness, and personality styles of their owners. Journal of Forensic Sciences, 57 (1), 152159.

Schwartz, R., Fremouw, W., Schenk, A. M., \& Ragatz, L. (2012). Psychological profile of male and female animal abusers. Journal of Interpersonal Violence, 27 (5), 846-861.

Ragatz, L., Schenk, A. M., \& Fremouw, W. (2011). Vicious dog ownership: Is it a thin slice of antisocial behavior? In R. Clarke (Ed.), Antisocial behavior: Causes, correlations, and treatments (pp. 1-12). Nova Science Publishers, Inc.

Fremouw, W. \& Schenk, A. M. (2010). Review of: The Cambridge Handbook of Forensic Psychology. Journal of Forensic Sciences, 56 (2), 567.

\section{POSTER PRESENTATIONS}

Keelan, C., Schenk, A. M., \& Fremouw, W. (2013, March). Empathy deficits among college cyber and traditional bullies? Poster presented at the Annual Meeting of the Southeastern Psychological Association, Atlanta, GA.

Patton, C., Schenk, A. M., Keelan, C., \& McNally, M. (2013, March). The interpersonal world of college bullies: Parents, peers, and partners. Poster presented at the Annual Meeting of the Southeastern Psychological Association, Atlanta, GA.

Schenk, A. M. \& Fremouw, W. (2012, March). A psychological profile of college cyberbullies. Poster session presented at the meeting of the American Psychology-Law Society, San Juan, PR.

Keelan, C., Cooper-Lehki, C., Schenk, A. M., \& Fremouw, W. (2012, March). Underreporting of adolescent bestiality: Polygraph vs. self-report measures. Poster session presented at the Eastern Psychological Association, Pittsburgh, PA.

Schenk, A. M., Ragatz, L., Fremouw, W., \& Keelan, C. (2011, March). Vicious dog ownership: A thin slice of antisocial personality? Poster session presented at the meeting of the American Psychology-Law Society, Miami, FL. 
Schenk, A. M. \& Fremouw, W. (2011, March). Psychological impact of cyberbully victimization among college students. Poster session presented at the meeting of the American Psychology-Law Society, Miami, FL.

Fremouw, W., Ragatz, L. L., Schwartz, R., Anderson, R., Schenk, A. M., \& Kania, K. (2010, March). Criminal thinking, aggression, and psychopathy in late high school bully-victims. Poster session presented at the meeting of the American Psychology-Law Society, Vancouver, BC.

Schwartz, R., Fremouw, W., Ragatz, L., \& Schenk, A. M. (2010, March). Psychological profile of male and female college-aged animal abusers versus controls. Poster session presented at the meeting of the American Psychology-Law Society, Vancouver, BC.

Schenk, A. M. \& Abbott, D. H. (2007, April). Digit Ratios as a Biomarker for Prenatal Androgen Excess. Poster presented at the University of Wisconsin-Madison Undergraduate Symposium, Madison, WI.

\section{TEACHING EXPERIENCE}

\section{Invited Guest Lecturer}

Fall 2013

Ethics and Professional Issues, Forest Institute of Professional Psychology

Presented lectures on APA's Ethical Principles of Psychologists and Code of Conduct to a graduate class.

- Taught a two-hour lecture on "Resolving Ethical Issues and Competence," and an additional two-hour lecture on "Human Relations" to a class of 18 graduate students

- Facilitated interactive discussions regarding important ethical issues encountered in the field of psychology

\section{Graduate Teaching Assistant}

\section{Psychology of Juvenile Offenders, West Virginia University}

Spring 2012

Designed and taught an upper-level, undergraduate psychology course focusing on juvenile delinquency and other forensically-related topics.

- Designed the course syllabus, which included the goals and learning objectives for students enrolled in the course, outlined graded activities and grading scale, and specified weekly assigned readings and class meeting topics

- Reviewed and selected a textbook on juvenile delinquency that best matched the specified learning objectives of this course

- Presented a weekly lecture for 2.5 hours to a class of 35 upper-level undergraduate students majoring in psychology, and graded all assignments with the course co-lecturer

- Constructed lectures and discussion questions to encourage in-class participation

Graduate Teaching Assistant Introduction to Psychology, West Virginia University

August 2009 - May 2010 
Taught Introduction to Psychology (Psych 101) to undergraduate students at West Virginia University.

- Prepared and presented two lectures each week to a class of 115 undergraduate students

- Attended weekly meetings to discuss effective teaching strategies to implement in the classroom

- Provided students with weekly office hours to clarify material, discuss grades, accommodate students with disabilities, and address any other issues

\section{SUPERVISIORY EXPERIENCE}

\section{Supervisor of Graduate Student Therapists Quin Curtis Center, West Virginia University}

August 2012-June 2013

Served as a mid-level supervisor on a treatment team providing outpatient anger management treatment.

- Instructed, modeled, and discussed different therapeutic techniques and skills with fellow graduate students

- Reviewed the notes and reports of the graduate student supervisee for accuracy and completeness

- Observed sessions directly and via video recording and provided supervisee with feedback on clinical techniques and skills, as well as offered treatment guidance to implement with the client

- Met as a team with a faculty supervisor to discuss clinically relevant issues, request feedback, and process sessions

\section{Supervisor of Research Assistants \\ October 2011-May 2011 Fremouw Forensic Psychology Research Lab, West Virginia University}

Supervised four undergraduate research assistants in hand-scoring a psychological measure

- Trained assistants how to score the Symptoms Checklist-90-Revised (SCL-90-R) and educated them about the utilization of the measure

- Organized a systematic scoring system to ensure all tasks were properly completed

- Completed quality assurance checks on $15 \%$ of all scored data

- Arranged monthly lab meetings to discuss research articles and topics in forensic psychology

\section{PROFESSIONAL AFFILIATIONS AND RECOGNITION}

American Psychological Association (APA)

American Psychology - Law Society, Division 41(AP-LS)

Eastern Psychological Association (EPA)

Golden Key National Honour Society

The National Scholars Honor Society

Psi Chi, Psychology National Honors Society 


\section{JOURNAL REVIEWS}

Invited reviewer for BMC Health Services Research Journal Invited reviewer for Jones and Bartlett Learning Publishers Guest reviewer for Journal of Aggression and Violent Behavior Guest reviewer for Journal of Forensic Sciences

\section{ADDITIONAL TRAININGS}

Dialectical Behavior Therapy Workshop (12 hours)

Domestic Violence 101: Understanding the Experience of Survivors (2 hours)

Ethics in a Correctional Setting ( 2 hours)

Forensic Case Law Seminar (1.5 hours/week for one year)

Malpractice and the Suicidal Client ( 3 hours)

MMPI-2-RF: Forensic Practice Briefing (2 hours)

Motivational Interviewing Workshop (6 hours)

Sex Offender Treatment and Assessment Training (8 hours)

\section{LEADERSHIP AND VOLUNTEER EXPERIENCE}

Elected APA Graduate Student Campus Representative August 2012 - July 2013

West Virginia University Psychology Department, Morgantown, WV

Volunteered with Learning \& Developmentally Delayed Children Jan. 2008 - Jan. 2013

Red Apple Elementary School, Racine, WI

Vice President

Psi Chi-Psychology National Honors Society, Madison, WI

Volunteer Relief Worker

Lutheran Disaster Response Trip, New Orleans, LA

August 2008 - May 2009

January 2007 